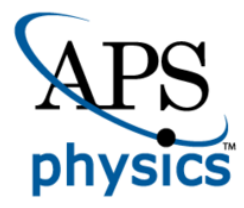

This is the accepted manuscript made available via CHORUS. The article has been published as:

\title{
Equation of state in $(2+1)$-flavor QCD
}

A. Bazavov, Tanmoy Bhattacharya, C. DeTar, H.-T. Ding, Steven Gottlieb, Rajan Gupta, P. Hegde, U. M. Heller, F. Karsch, E. Laermann, L. Levkova, Swagato Mukherjee, P. Petreczky,

C. Schmidt, C. Schroeder, R. A. Soltz, W. Soeldner, R. Sugar, M. Wagner, and P. Vranas (HotQCD Collaboration)

Phys. Rev. D 90, 094503 - Published 4 November 2014 DOI: 10.1103/PhysRevD.90.094503 


\title{
The equation of state in $(2+1)$-flavor QCD
}

\author{
A. Bazavov ${ }^{a}$, Tanmoy Bhattacharya ${ }^{\mathrm{b}}$, C. DeTar ${ }^{\mathrm{c}}$, H.-T. Ding ${ }^{\mathrm{d}}$, Steven Gottlieb ${ }^{\mathrm{e}}$, Rajan Gupta ${ }^{\mathrm{b}}$, P. Hegde ${ }^{\mathrm{d}}$, \\ U.M. Heller ${ }^{\mathrm{f}}$, F. Karsch ${ }^{\mathrm{g}, \mathrm{h}}$, E. Laermann ${ }^{\mathrm{h}}$, L. Levkovac ${ }^{\mathrm{c}}$, Swagato Mukherjeeg, P. Petreczkyg,

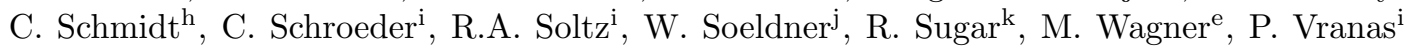

(HotQCD Collaboration)

\author{
a Department of Physics and Astronomy, \\ University of Iowa, Iowa City, IA 52240, USA \\ b Theoretical Division, Los Alamos National Laboratory, \\ Los Alamos, NM 87545, USA \\ c Department of Physics and Astronomy, \\ University of Utah, Salt Lake City, UT 84112, USA \\ d Key Laboratory of Quark \& Lepton Physics (MOE), \\ Institute of Particle Physics, \\ e Physics Department, Indiana University, \\ Bloomington, IN 47405, USA \\ f American Physical Society, \\ One Research Road, Ridge, NY 11961, USA \\ g Physics Department, Brookhaven National Laboratory, \\ Upton, NY 11973, USA \\ h Fakultät für Physik, Universität Bielefeld, \\ D-33615 Bielefeld, Germany \\ i Physics Division, Lawrence Livermore National Laboratory, \\ Livermore CA 94550, USA \\ j Institut für Theoretische Physik, \\ Universität Regensburg, D-93040 Regensburg, Germany \\ k Physics Department, University of California, \\ Santa Barbara, CA 93106, USA
}

\begin{abstract}
We present results for the equation of state in $(2+1)$-flavor QCD using the highly improved staggered quark action and lattices with temporal extent $N_{\tau}=6,8,10$, and 12 . We show that these data can be reliably extrapolated to the continuum limit and obtain a number of thermodynamic quantities and the speed of sound in the temperature range (130-400) MeV. We compare our results with previous calculations, and provide an analytic parameterization of the pressure, from which other thermodynamic quantities can be calculated, for use in phenomenology. We show that the energy density in the crossover region, $145 \mathrm{MeV} \leq T \leq 163 \mathrm{MeV}$, defined by the chiral transition, is $\epsilon_{c}=(0.18-0.5) \mathrm{GeV} / \mathrm{fm}^{3}$, i.e., $(1.2-3.1) \epsilon_{\text {nuclear. }}$. At high temperatures, we compare our results with resummed and dimensionally reduced perturbation theory calculations. As a byproduct of our analyses, we obtain the values of the scale parameters $r_{0}$ from the static quark potential and $w_{0}$ from the gradient flow.
\end{abstract}

PACS numbers: 11.15.Ha, 12.38.Gc, 12.38Mh

\section{INTRODUCTION}

At high temperatures, matter governed by strong interactions (strong interaction matter) undergoes a deconfining transition to a new state, in which the thermodynamics can be described in terms of quark and gluon degrees of freedom. The equation of state (EoS) of such matter, just as for many other thermodynamic systems, is of fundamental importance for understanding its composition as well as its static and dynamical properties. Studying the properties of this matter using Quantum ChromoDynamics (QCD) was made possible by the formulation of lattice-regularized QCD [1] and the development of numerical algorithms for its analysis [2]. Lattice calcula- tions of the QCD EoS were first performed in 1980 [3], and, driven by the steady growth in computing resources and the development of new simulation algorithms, there now exist precise results for the transition temperature $[4,5]$, fluctuations of conserved charges [6-8] as well as the EoS. For recent reviews see for instance Refs. [9-11].

The EoS contains information on the relevant degrees of freedom in the thermal medium in different temperature regimes and reflects the transition between different states of matter. A quantitative description of the QCD EoS over a wide temperature range is needed to understand the expansion and cooling of matter in the early universe, as well as of the hot dense nuclear matter created in heavy ion collisions. 
To study the QCD EoS across a transition between different states of matter, at which the internal degrees of freedom are highly correlated, requires nonperturbative techniques. However, in the case of strong interaction matter, the need for nonperturbative methods is not restricted to the strongly interacting region close to the QCD transition temperature, but is also needed far above this deconfining transition where well-known infrared problems [12] prohibit a straightforward perturbative analysis of QCD thermodynamics. Also, at low temperatures, where the hadron resonance gas models (HRG) for the description of the hadronic equation of state are quite successful [13], lattice QCD calculations are important as they provide the benchmark estimates of thermal properties of in-medium hadrons and the EoS of hadronic matter. In summary, simulations of lattice QCD provide the best approach over the full phenomenologically interesting temperature range in which all sources of errors can be quantified and systematically improved.

The deconfining transition in QCD, with small but non-zero values of the light quark masses, is a rapid crossover that coincides with the restoration of chiral symmetry $[4,5]$. In fact, it is the latter that characterizes the second order phase transition that occurs in the chiral limit of QCD at finite temperature. At this phase transition the spontaneously broken chiral symmetry is restored. The universal scaling properties of this chiral transition are used to determine the pseudo-critical temperature $T_{c}$ at which the rapid crossover with the physical light and strange quark masses takes place [5]. Extensive simulations of lattice QCD at zero net baryon number density have established that this crossover transition occurs at $T_{c} \sim 155 \mathrm{MeV}$ for the physical spectrum of two light and a heavier strange quark $[4,5]$. Even though there is no well-defined separation of phases because of the crossover nature of the transition, it is well established that many thermodynamic properties change rapidly in the vicinity of $T_{c}$. Along with the analysis of fluctuations in the chiral condensate that are used to probe the restoration of chiral symmetry and to determine $T_{c}$, the study of fluctuations in conserved charges provides clear evidence for deconfinement of light and strange quark degrees of freedom, i.e., a transition from hadronic to quark-gluon degrees of freedom around $T_{c}[14]$.

In this paper, we present a detailed analysis of the EoS that captures the crossover transition and the temperature range that is relevant to the hydrodynamic evolution of heavy ion collisions at the Relativistic Heavy Ion Collider (RHIC) and the Large Hadron Collider (LHC). We performed high statistics simulations of lattice QCD on lattices of size $N_{\sigma}^{3} N_{\tau}$ for four values of $N_{\tau} \equiv(a T)^{-1}=6$, 8,10 , and 12 and a large spatial size $N_{\sigma}=4 N \tau$. We use these data to show that a controlled extrapolation to the continuum can be performed in the temperature range $130 \mathrm{MeV} \leq T \leq 400 \mathrm{MeV}$. We also show that the rapid change in the energy density signaling the lib- eration of quark-gluon degrees of freedom leads to an estimate of the pseudo-critical temperature that is consistent with that obtained from the analysis of chiral symmetry restoration. Lastly, we provide an accurate parametrization of this EoS that can be used for hydrodynamic modeling of heavy ion collisions (see Ref. [15] for a recent review) and other phenomenological studies of the thermodynamics of strong interaction matter.

Most of the lattice QCD calculations of the thermodynamics of strong interaction matter use the staggered fermion discretization scheme. The main reason for this is that staggered fermions preserve an essential remnant of the continuum $S U(2)_{L} \times S U(2)_{R}$ chiral symmetry of the light quark sector and are, at the same time, the least demanding computationally. For an overview of EoS calculations using other fermion discretization schemes see Refs. [10, 16]. Furthermore, simulations of QCD thermodynamics using staggered fermions have been systematically improved by eliminating $\mathcal{O}\left(a^{2}\right)$ cutoff effects [17] and reducing the effects of the so-called taste symmetry breaking, specific to the staggered fermion formulation, by using smeared gauge links $[18,19]$.

A number of improved staggered formulations have been developed and used to study QCD at finite temperature. In the past, we have simulated the $\mathrm{p} 4$ and asqtad actions [20-24]. These actions eliminate tree-level $\mathcal{O}\left(a^{2}\right)$ cutoff effects on lattices with moderate, $N_{\tau}>8$, lattices but have large taste symmetry violations at low temperatures. The Wuppertal-Budapest collaboration has used the stout-smeared staggered action [25] that very effectively reduces taste symmetry violation effects but still shows large $\mathcal{O}\left(a^{2}\right)$ cutoff effects at high temperatures. The first reliable continuum extrapolated results for the QCD EoS have recently been obtained with this action [26].

The calculations presented in this paper are carried out using the highly improved staggered quark (HISQ) action introduced by the HPQCD collaboration [27]. It was designed to improve both the taste symmetry and the quark dispersion relation by including smeared onelink terms as well as straight three-link terms that completely eliminate $\mathcal{O}\left(a^{2}\right)$ discretization errors at tree level. The HISQ action has turned out to yield the smallest violations of taste symmetry among the currently used staggered fermion actions $[5,28,29]$. We have used it extensively to carry out high precision studies of the chiral and deconfinement aspects of the QCD transition which lead to the estimate $T_{c}=154(9) \mathrm{MeV}$ for the QCD transition temperature. It has also been used to study the fluctuations of conserved charges [7,30-32] and various spatial and temporal correlation functions [33, 34]. The study of fluctuations of conserved charges at high temperatures demonstrates, in particular, that the HISQ action is indeed very effective in reducing cutoff effects [32].

In this paper, we show that continuum extrapolated results for the EoS of $(2+1)$-flavor QCD obtained with the HISQ action are in good agreement with those ob- 
tained with the stout action $[25,26]^{1}$. There are, however, systematic differences which may start to become of relevance in the analysis of the approach to the perturbative limit at high temperatures. We will discuss these features in more detail in Secs. IV and V.

The rest of the paper is organized as follows. In Sec. II we discuss the lattice setup and the simulation parameters. Section III contains the results for the trace anomaly, which is the basic thermodynamic quantity obtained from lattice calculations, and from which the EoS is obtained. Section IV discusses the extraction of thermodynamic quantities in the continuum limit. This section ends with an analytical parameterization of the EoS that matches the HRG estimates below $T=130 \mathrm{MeV}$ and the lattice data between 130 and $400 \mathrm{MeV}$. In Sec. $\mathrm{V}$, we present results on observables that depend on second order derivatives of the pressure with respect to temperature, i.e., the specific heat and the speed of sound. We discuss their phenomenological importance. Also in Sec. V, we discuss how our results for the EoS connect to high temperature perturbative calculations. Finally, Sec. VI contains our conclusions. Technical details of the calculations are given in the appendices.

\section{LATTICE SETUP}

We performed simulations of $(2+1)$-flavor QCD using the HISQ action and the tree-level improved gauge action. This combination is referred to as the HISQ/tree action. The $(2+1)$-flavor simulations are defined by three bare parameters, the gauge coupling $\beta=10 / g^{2}$, the lightquark mass $m_{l}=m_{u}=m_{d}$, and the heavier strange quark mass $m_{s}$. For a given value of the gauge coupling, we tune the strange quark mass to its physical value by matching the mass of the fictitious unmixed pseudoscalar $\eta_{s \bar{s}}$ meson to $695 \mathrm{MeV}$. The light quark mass is fixed as a fraction of the strange quark mass, $m_{l}=m_{s} / 20$. This is slightly above the physical ratio $m_{l}=m_{s} / 27.3$ and corresponds to a pion mass of about $160 \mathrm{MeV}$ in the continuum limit. This difference should, however, give rise to negligible effects in the calculation of the EoS [24, 25]. Having fixed $m_{s}$ and $m_{l}$, the continuum limit is taken along a line of constant physics (LCP) controlled by a single parameter, the gauge coupling $\beta$.

The LCP for the HISQ/tree action and $m_{l}=m_{s} / 20$ has been established, and reported in Ref. [5], based on a set of zero-temperature ensembles that span the range of gauge couplings $\beta=5.9-7.28$. In that study, the associ-

\footnotetext{
1 There was an error in the preliminary analyses of the EoS with the HISQ/tree action presented in conference proceedings before 2014 [35-37] due to an incorrect normalization of the fermion contribution to the trace anomaly. This error gave a larger value of the trace anomaly for $T<300 \mathrm{MeV}$. Preliminary results for the EoS with the HISQ/tree action prior to 2014 are, therefore, superceded.
}

ated sets of finite temperature ensembles were generated on lattices with temporal extent $N_{\tau}=6,8$, and 12 , and a fixed aspect ratio $N_{\sigma} / N_{\tau}=4$ for the spatial extent. In Appendix A, we list all the zero- and finite-temperature gauge field ensembles used in this study along with the final statistics. Here we briefly summarize the additional simulations carried out, and the improvements made, compared to those presented in Ref. [5].

(i) Additional zero- and finite-temperature ensembles were generated at $\beta=7.373,7.596$, and 7.825.

(ii) A new set of finite temperature lattices with $N_{\tau}=$ 10 were generated.

(iii) The statistics are substantially increased for all existing ensembles, in some cases by more than an order of magnitude compared to Ref. [5].

(iv) The determination of the LCP on finer lattices is improved by measurements of the static quark potential and the hadron spectrum on new zerotemperature ensembles.

The lattice spacing $a$, corresponding to the coupling $\beta$, was determined by calculating the scales $r_{0}$ [38] and $r_{1}$ [39], defined in terms of the static potential as

$$
\left.r^{2} \frac{d V}{d r}\right|_{r_{i}}=C_{i}, \quad i=0,1
$$

where $C_{0}=1.65$ and $C_{1}=1.0$. At each $\beta$, these scales are determined by first extracting the potential $V(r)$ by fitting the lattice data to

$$
V(r)=C+\frac{B}{r}+\sigma r,
$$

and then calculating its derivative in intervals around the values of $r_{1}$ and $r_{0}$, as described in Ref. [5]. The details of the determination of $r_{0} / a$ and $r_{1} / a$ and the extrapolation of the ratio, $r_{0} / r_{1}$, to the continuum limit are given in Appendix B. The extrapolated result is $r_{0} / r_{1}=1.5092(39)$, which gives $r_{0}=0.4688(41) \mathrm{fm}$ using the physical value $r_{1}=0.3106(14)(8)(4) \mathrm{fm}$ [40]. This estimate of $r_{0}$ is in agreement with $r_{0}=0.48(1)(1) \mathrm{fm}$ given in Ref. [4].

To crosscheck the precision of the determination of the lattice spacing, we also calculated the scale $w_{0}$ first proposed in Ref. [41]. The details of this calculation are also given in Appendix $\mathrm{B}$, and we obtain $w_{0} / r_{1}=0.5619(21)$ in the continuum limit. This translates to $w_{0}=0.1749(14) \mathrm{fm}$, in agreement with $w_{0}=$ $0.1755(18)(4) \mathrm{fm}$ given in Ref. [41].

We have also measured the masses and decay constants of several light hadrons. These allow us to improve the determination of the LCP at weaker coupling and provide further crosschecks on the scale setting in the continuum limit. We find that the different ways to set the lattice scale using hadronic observables agree with each other and the scale determined using $r_{1}$ within the estimated errors. The details of these analyzes are presented in Appendix C. 


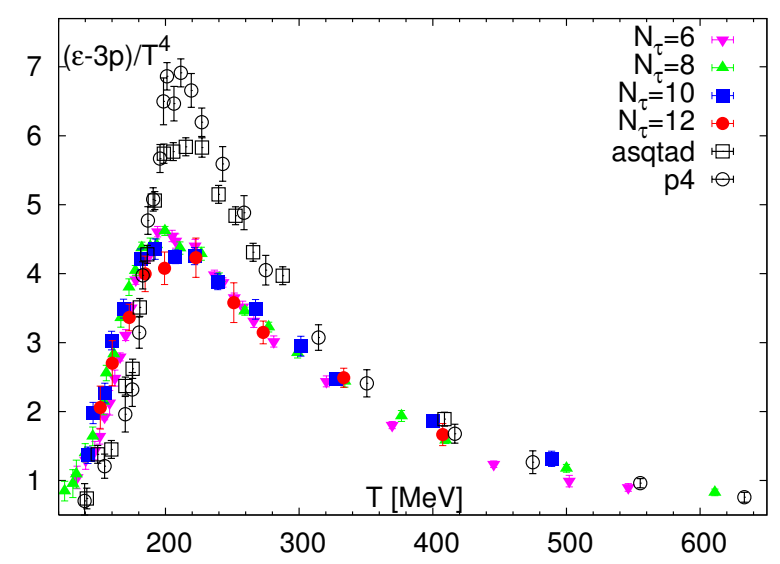

Figure 1: The trace anomaly calculated with the HISQ/tree action at different $N_{\tau}$ and compared with results from previous calculations with the p4 and asqtad actions on $N_{\tau}=8$ lattices [23], except for the two highest temperatures, where we show the $N_{\tau}=6 \mathrm{p} 4$ data from Ref. [42] and Ref. [22], respectively.

\section{THE TRACE ANOMALY}

The QCD partition function on a hypercubic lattice of size $N_{\sigma}^{3} N_{\tau}$, after integration over the fermion degrees of freedom, is given by

$$
Z\left(\beta, N_{\sigma}, N_{\tau}\right)=\int \prod_{x, \mu} d U_{x, \mu} e^{-S(U)},
$$

where $U_{x, \mu} \in S U(3)$ are the gauge field variables, labeled by $x$ and $\mu$, defined on the links between lattice points and the Euclidean action $S(U)$ is the sum of the gauge and fermionic parts:

$$
S(U)=\beta S_{G}(U)-S_{F}(U) .
$$

The temperature in physical units is set by the temporal extent $N_{\tau}$ of the lattice and related to the lattice spacing $a$ as $T=1 /\left(a N_{\tau}\right)$.

The trace of the energy-momentum tensor, also called trace anomaly or the interaction measure, is related to the pressure $p$ as (see Ref. [22])

$$
\frac{\Theta^{\mu \mu}(T)}{T^{4}}=\frac{\epsilon-3 p}{T^{4}}=T \frac{d}{d T}\left(\frac{p}{T^{4}}\right),
$$

with $\epsilon$ denoting the energy density. $\Theta^{\mu \mu}(T)$ can be defined on the lattice as the total derivative of $\ln Z$ with respect to the lattice spacing $a$ :

$$
\Theta^{\mu \mu}=\epsilon-3 p=-\frac{T}{V} \frac{d \ln Z}{d \ln a} .
$$

The right hand side of Eq. (6) is straightforward to evaluate on the lattice and gives

$$
\frac{\epsilon-3 p}{T^{4}} \equiv \frac{\Theta_{G}^{\mu \mu}(T)}{T^{4}}+\frac{\Theta_{F}^{\mu \mu}(T)}{T^{4}},
$$

$$
\begin{gathered}
\frac{\Theta_{G}^{\mu \mu}(T)}{T^{4}}=R_{\beta}\left[\left\langle s_{G}\right\rangle_{0}-\left\langle s_{G}\right\rangle_{\tau}\right] N_{\tau}^{4} \\
\frac{\Theta_{F}^{\mu \mu}(T)}{T^{4}}=-R_{\beta} R_{m}\left[2 m_{l}\left(\langle\bar{\psi} \psi\rangle_{l, 0}-\langle\bar{\psi} \psi\rangle_{l, \tau}\right)\right. \\
\left.+m_{s}\left(\langle\bar{\psi} \psi\rangle_{s, 0}-\langle\bar{\psi} \psi\rangle_{s, \tau}\right)\right] N_{\tau}^{4} .
\end{gathered}
$$

Here $\left\langle s_{G}\right\rangle_{\tau(0)}$ is the expectation value of the action density for the gauge fields evaluated at finite (zero) temperature and $\langle\bar{\psi} \psi\rangle_{l(s), \tau(0)}$ stands for the expectation values of light $(l)$ and strange $(s)$ quark chiral condensates evaluated at finite (zero) temperature. Subtracting the zero temperature values in the above expressions ensures that all thermodynamic quantities are finite in the continuum limit. In Eq. (9), we have used the single flavor normalization for both the light and strange quark condensates as in previous works $[5,22,23]$. The nonperturbative beta function and mass renormalization function are defined as $[22,23]$

$$
\begin{aligned}
& R_{\beta}(\beta)=\frac{r_{1}}{a}\left(\frac{\mathrm{d}\left(r_{1} / a\right)}{\mathrm{d} \beta}\right)^{-1}, \\
& R_{m}(\beta)=\frac{1}{m_{s}(\beta)} \frac{\mathrm{d} m_{s}(\beta)}{\mathrm{d} \beta} .
\end{aligned}
$$

The determination of these functions is discussed in Appendices B-D. In the above equations, we explicitly separated the contributions to the trace anomaly that come from purely gluonic operators $\Theta_{G}^{\mu \mu}(T)$ and fermionic operators $\Theta_{F}^{\mu \mu}(T)$. Even though we will refer to them as the gluonic and fermionic parts, it would be misleading to consider $\Theta_{F}^{\mu \mu}(T)$ as the quark contribution to the trace anomaly. For example, for massless quarks $\Theta_{F}^{\mu \mu}(T) / T^{4}$ is zero, while massless quarks certainly contribute to the trace anomaly. At high temperatures, where the effect of nonzero quark masses is expected to be small, the quark contribution almost exclusively comes from $\Theta_{G}^{\mu \mu}(T)$. As we will see below, this expectation is confirmed by our numerical data. The above separation of the trace anomaly into $\Theta_{G}^{\mu \mu}(T)$ and $\Theta_{F}^{\mu \mu}(T)$ is, however, useful in the analysis of lattice data as they are expected to be affected differently by the taste symmetry breaking inherent in staggered fermions and because the statistical errors are also different.

The pressure can be calculated using the integral method, i.e., by inverting Eq. (5):

$$
\frac{p(T)}{T^{4}}=\frac{p_{0}}{T_{0}^{4}}+\int_{T_{0}}^{T} d T^{\prime} \frac{\Theta^{\mu \mu}}{T^{\prime 5}} .
$$

The choice of the reference temperature $T_{0}$ and pressure $p_{0}$ is discussed in Sec. IV. All other thermodynamic quantities, defined as appropriate derivatives of the partition function with respect to the temperature, can be calculated from Eqs. (5) and (12) by using standard thermodynamic identities.

Since the trace anomaly is the central quantity in the lattice calculations of the EoS, we discuss its properties 

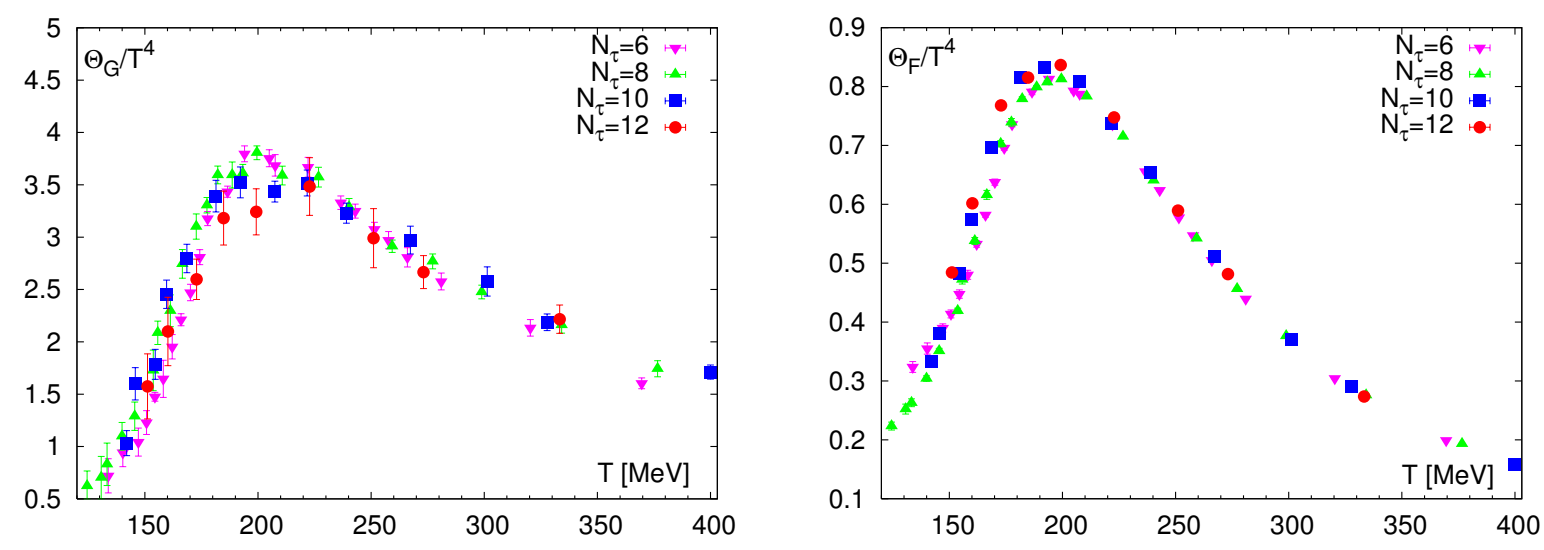

Figure 2: The gluonic (left) and fermionic (right) parts of the trace anomaly for different $N_{\tau}$. See text for details.

in some detail. In Fig. 1, we compare results for the trace anomaly obtained with the HISQ/tree action on lattices with temporal extent $N_{\tau}=6,8,10$, and 12 with our previous findings using the $\mathrm{p} 4$ and asqtad actions [22, 23, 42]. The cutoff effects are much smaller in the HISQ/tree action and the height of the peak is significantly reduced. Below the peak, the HISQ/tree data are larger than the p4 and asqtad results, but significantly smaller at temperatures around and higher than the peak. These large deviations reflect the fact that the asqtad and the p4 actions have much larger cutoff effects at low temperatures and in the crossover region (see discussions in Ref. [5]). The smaller taste violations of the HISQ action lead to a smaller root-mean-square mass in the pseudoscalar sector [5], i.e., to a smaller average pion mass, which leads to a larger trace anomaly as well as larger pressure and energy density in the low temperature, hadronic region. For $T>350 \mathrm{MeV}$, we find reasonably good agreement between the results obtained with different actions. This is, to some extent, expected as at such high temperatures, i.e., at small $a$, all the above actions should have small cutoff effects. This expectation has been demonstrated in the calculations of quark-number susceptibilities [32]. In Sec. IV, we show that having small cutoff effects in the data with the HISQ/tree action allows us to make robust continuum extrapolations and obtain a precise EoS in the temperature range 130-400 $\mathrm{MeV}$.

A closer look at the HISQ/tree action data shown in Figs. 1, 2, and 3 reveals some cutoff effects at low temperatures and in the peak region. It is instructive to discuss these cutoff effects separately in terms of the gluonic, $\Theta_{G}^{\mu \mu}$, and the fermionic, $\Theta_{F}^{\mu \mu}$, contributions defined in Eqs. (8) and (9), respectively, and shown in Fig. 2. We find that the trace anomaly is dominated by the gluonic part. The fermionic contribution is about $(20-25) \%$ of the gluonic contribution in the peak region, rises to $\sim 35 \%$ below it, and becomes much smaller at high temperatures. At $T=400 \mathrm{MeV}$, it is only about $10 \%$. Around the peak, $\Theta_{G}^{\mu \mu}$, and consequently the trace anomaly, shows a decrease with increasing $N_{\tau}$, i.e., the continuum limit is approached from above.

The statistical errors and the lattice discretization effects in the HISQ/tree data are smaller in the fermionic part compared to the gluonic part. In $\Theta_{F} / T^{4}$, we observe significant cutoff effects only at the lowest temperature $T \sim 133 \mathrm{MeV}$, where the $N_{\tau}=6$ and 8 data differ by about $30 \%$. This small size of cutoff effects in $\Theta_{F}^{\mu \mu}$ with the HISQ/tree action in the low temperature region is in contrast to results obtained using the asqtad and the p4 actions, where the fermionic part showed significantly larger cutoff effects. We also note that cutoff effects arising from taste symmetry violations have opposite effects in $\Theta_{F}^{\mu \mu}$ and $\Theta_{G}^{\mu \mu}$. While a larger root-mean-square (RMS) mass for the pions leads to smaller values of $\Theta_{G}^{\mu \mu}$ at low temperatures, it leads to larger values in $\Theta_{F}^{\mu \mu}$ as the chiral condensates are larger for larger pion masses.

In the total trace anomaly, significant discretization effects are observed only in the peak and low-temperature regions. Within errors, we find no cutoff effects on comparing $N_{\tau}=6,8$, and 10 , data for $T<145 \mathrm{MeV}$. In the interval $145 \mathrm{MeV}<T<170 \mathrm{MeV}$, we observe some cutoff dependence, with the largest difference between the $N_{\tau}=6$ and 8 data.

At low temperatures, all thermodynamic quantities are expected to be well-described by the hadron resonance gas (HRG) model, in which all the hadrons and hadron resonances are assumed to contribute to the thermodynamics as non-interacting particles. Many previous studies have confirmed this expectation $[6,7,31,43-46]$. The trace anomaly in the HRG model is given by

$$
\begin{gathered}
\left(\frac{\epsilon-3 p}{T^{4}}\right)^{H R G}= \\
\sum_{m_{i} \leq m_{\max }} \frac{d_{i}}{2 \pi^{2}} \sum_{k=1}^{\infty} \frac{\left(-\eta_{i}\right)^{k+1}}{k}\left(\frac{m_{i}}{T}\right)^{3} K_{1}\left(\frac{k m_{i}}{T}\right)
\end{gathered}
$$

where different particle species of mass $m_{i}$ have degeneracy factors $d_{i}$ and $\eta_{i}=-1(+1)$ for bosons (fermions). The particle masses are taken from the Particle Data Book [47], including all known states up to the resonance 


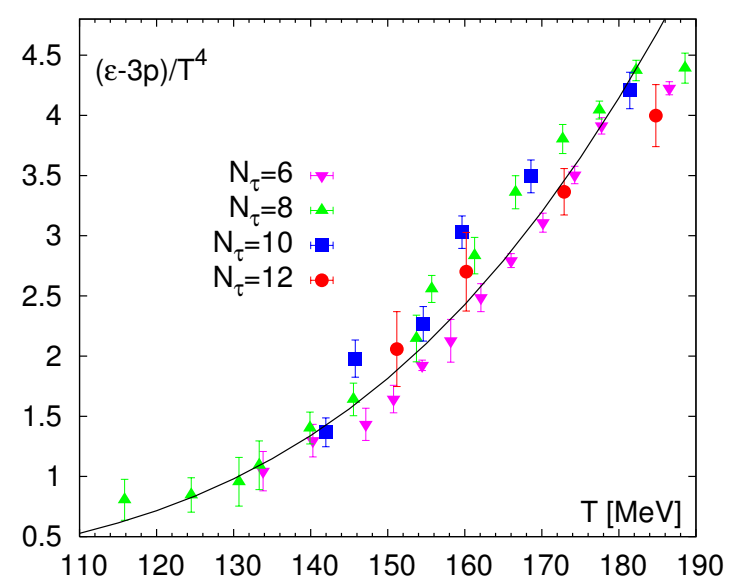

Figure 3: The trace anomaly in the low temperature region compared with the hadron resonance gas model (solid line).

mass of $m_{\max }=2.5 \mathrm{GeV}$. We compare the predictions of the HRG model with our data for the trace anomaly in the low temperature region in Fig. 3. For $T<145 \mathrm{MeV}$ the lattice data do not show any significant $N_{\tau}$ dependence and are in good agreement with estimates from the HRG model. This agreement will be used in an important way for the continuum extrapolation and for the calculation of the pressure described in the next section. For temperatures in the interval $145 \mathrm{MeV}<T<170 \mathrm{MeV}$, the $N_{\tau}=8,10$ and 12 lattice data lie above the HRG curve, while the $N_{\tau}=6$ data lie systematically below. In Sec. IV, we show that the cutoff effects in the $N_{\tau}=6$ data are large in this temperature interval.

\section{THERMODYNAMICS IN THE CONTINUUM LIMIT}

In this section, we describe the calculation of the pressure and the energy and entropy densities in the continuum limit. The main step in this calculation is the extrapolation of the lattice data for the trace anomaly $\Theta^{\mu \mu}$ from $N_{\tau}=6,8,10$, and 12 lattices to the continuum limit. Noting that the leading lattice discretization effects $\left(N_{\tau}\right.$ dependence) for staggered fermions are expected to be proportional to $(a T)^{2} \sim 1 / N_{\tau}^{2}$, we use the fit ansatz

$$
\frac{\Theta^{\mu \mu}(T)}{T^{4}}=A+\sum_{i=1}^{n_{k}+3} B_{i} \times S_{i}(T)+\frac{C+\sum_{i=1}^{n_{k}+3} D_{i} \times S_{i}(T)}{N_{\tau}^{2}},
$$

where $n_{k}$ denotes the number of knots in the interior of the fit interval and the $S_{i}$ are a set of basis cubic splines with discontinuities only in the third derivative at the specified knots as described below. ${ }^{2}$ The positions of the knots and the constants $A, B_{i}, C$, and $D_{i}$ are parameters that are determined by the fit. To test whether it is sufficient to keep just the leading $1 / N_{\tau}^{2}$ term, we also considered the next, $O\left(1 / N_{\tau}^{4}\right)$, correction,

$$
\frac{E+\sum_{i=1}^{n_{k}+3} F_{i} \times S_{i}(T)}{N_{\tau}^{4}} .
$$

Adding these terms to the quadratic fit, given in Eq. (14), defines the quartic fit also discussed below.

The basic assumption underlying the proposed fit ansatz is that the data and the variation with $N_{\tau}$ can be described by a set of piecewise continuous splines of cubic order. The temperature interval to be fitted is divided into sub-regions by a finite number of internal knots $n_{k}$, which we further assume are independent of $N_{\tau}$. The number and position of these knots specify a set of basis splines that forms a complete set over the full interval, i.e., any piecewise continuous cubic function can be fitted by them. The number of knots needed depends, in general, on the complexity of the data; and the total number of basis splines invoked by the fit depends on the number of knots specified. The positions of the knots are outputs of the least-square minimization procedure we use.

A number of choices need to be specified before we can discuss the fits.

(i) The errors in each data point for $\Theta^{\mu \mu}(T) / T^{4}$ are assumed to be normally distributed and independent, since these come from independent simulations.

(ii) The entire analysis is done within a bootstrap procedure using 20,001 samples. This number was chosen to make the sampling error in the bootstrap estimate of the standard error $1 \%$. The bootstrap samples were generated by selecting each data point from a normal distribution with its width given by the quoted error. The final error band for $\Theta^{\mu \mu}(T) / T^{4}$ is given by the $1 \sigma$ spread of the bootstrap values at each temperature. The statistical package $\mathrm{R}$ [48-50] was used to implement this analysis.

(iii) The values of temperature at which simulations have been done are not uniform, in particular we do not have much data on $N_{\tau}=10$ and 12 lattices for $T<130 \mathrm{MeV}$ and $T>400 \mathrm{MeV}$. Our results will, therefore, be restricted to the range $130 \mathrm{MeV} \leq T \leq 400 \mathrm{MeV}$.

(iv) Our goal is to use the minimum number of knots, and thus, the minimum number of parameters. We

\footnotetext{
2 Note that when knots are coincident, successively lower derivatives are discontinuous. All the splines are defined to go to zero at the lower end of the fit interval as we explicitly include the constants $\mathrm{A}$ and $\mathrm{C}$ in our fit ansatz.
} 
studied the $\chi^{2}$ resulting from the least-square minimization procedure to settle on the number of knots.

(v) We analyze the data using both the quadratic and quartic ansatz and with and without the $N_{\tau}=6$ data. Our final results are obtained using the quadratic fit without the $N_{\tau}=6$ data.

(vi) The data on $N_{\tau}=8$ lattices for $T \leq 130 \mathrm{MeV}$ are insufficient to constrain the fits at the lower end. We, therefore, use the estimate $\Theta^{\mu \mu}(T) / T^{4}=1.007$ with slope 0.032 at $T=130 \mathrm{MeV}$, obtained from the HRG model, for the continuum extrapolated value. To justify this choice we note that the HRG model is a good approximation at this temperature and insensitive to possible higher resonances missed in the hadron spectrum [8]. Indeed, we find that the lattice data and the HRG estimates agree for $T<145 \mathrm{MeV}$. To take into account the uncertainty in the HRG estimates, both the estimate and the slope were picked using a Gaussian distribution about their central values with a conservatively chosen width, $10 \%$ of their respective values. We implemented this constrain by replacing the spline $B_{1}$ by a term proportional to $T-130 \mathrm{MeV}$ with its coefficient given by the HRG value. This constraint, therefore, reduces the number of free parameters in Eq. (14) by two.

(vii) The data on $N_{\tau}=8$ lattices in the temperature range (400-610)/ $\mathrm{MeV}$ was used to stabilize the quadratic fits up to $400 \mathrm{MeV}$. For the quartic fits, both the $N_{\tau}=6$ and 8 data at $T>400 \mathrm{MeV}$ were used.

To decide on the number of knots to use, we fit the $N_{\tau}=8,10$, and 12 data with the quadratic ansatz with 2-4 internal knots. The fit with two knots was the most stable and the $\chi^{2}$ did not improve significantly with additional knots. The choice of two knots is consistent with the observation that the data show three main regions: the low temperature region $T \lesssim 175 \mathrm{MeV}$, the peak region $(175-225 \mathrm{MeV})$ and the high temperature region $T \gtrsim 225 \mathrm{MeV}$. The fit parameters and the location of the knots are outputs of the $\chi^{2}$ minimization procedure. This fit has 53 data points, 5 basis splines and 12 free parameters, i.e., the 10 parameters remaining in Eq. (14) after imposing the HRG value and slope at $T=130 \mathrm{MeV}$ and the locations of the two knots. This ansatz fits all the data, and the $\chi^{2} /$ dof $=0.9$ for dof $=41$ was well distributed, i.e., it was not dominated by a few points nor by any one of the three regions. The distribution of the positions of the knots over the 20,001 samples had central values of $170 \mathrm{MeV}$ and $229 \mathrm{MeV}$ with a standard deviation of $8 \mathrm{MeV}$. This fit, called the final fit, is used for our continuum results as the tests itemized below did not improve upon it:

(i) Adding more knots to the final fit did not improve the fit. The additional parameters were poorly determined, and in most bootstrap samples two or more knots were coincident.

(ii) We added the $N_{\tau}=6$ data to the final fit. The $\chi^{2} /$ dof increased and the fit became skewed. It adjusted to preferentially fit the low error $N_{\tau}=6$ points and the $\chi^{2}$ became dominated by the $N_{\tau}=$ 12 data below the peak. We concluded that the quadratic ansatz is insufficient to fit the data at all four $N_{\tau}$ values.

(iii) We explored the quartic ansatz to fit the data at all four $N_{\tau}$ values. In this case, the best fit required three knots. The resulting error band overlaps with that of the the final fit except in the peak region, where it is about $1 \sigma$ lower. The position of the knots are not as stable as in the final fit, and in many bootstrap samples, two knots were coincident. To summarize, the final quadratic fit was preferred over the quartic fit as it is based on data closer to the continuum limit, has the least number of parameters and fits the data well as shown in Fig. 4.

The quality of the final fit using Eq. (14) is demonstrated in Fig. 4 where we show that the bootstrap error bands of the final fit describe the $N_{\tau}=8,10$, and 12 data very well. On the other hand, as stated previously, we find that the $N_{\tau}=6$ data lie outside the range of applicability of the quadratic ansatz. The same error bands are compared with the final continuum extrapolated result (black band) in Fig. 5.

Having determined the final fit, we obtained the pressure $p / T^{4}$ by numerically integrating the bootstrap samples for $\Theta^{\mu \mu}(T)$ between $130 \mathrm{MeV}$ and $400 \mathrm{MeV}$ using Eq. (12). For the integration constant $p_{0}$, the pressure at $T=130 \mathrm{MeV}$, we picked a value from a normal distribution with the mean value $p_{0} / T_{0}^{4}=0.4391$, again taken from the HRG model, and width 0.0439 , a conservative $10 \%$ error estimate on this HRG value. Since the estimate of $p_{0} / T_{0}^{4}$ is independent of the calculation of $\Theta^{\mu \mu}(T)$, this choice effectively adds a $\delta p_{0}$ in quadrature to the errors from integrating $\Theta^{\mu \mu}(T) / T^{4}$. Knowing $\Theta^{\mu \mu}(T) / T^{4} \equiv(\epsilon-3 p) / T^{4}$ and $p / T^{4}$, it is straightforward to derive the energy density, $\epsilon$, and the entropy density $s=(\epsilon+p) / T$.

The final systematic error that is folded into the estimates of all the thermodynamic quantities is the uncertainty in the determination of the lattice scale $a$, and thus the values of the temperature $T$ used in the fits. Based on the uncertainty analyses in the determination of the lattice scale $a(\sim 1.3 \%)$ and tuning of the $m_{s}$ to stay on the LCP presented in Appendices B and C, we assigned an overall conservative $2 \%$ uncertainty in $T$, which we add linearly to the error estimates already assigned by the bootstrap process. In practice, at each $T$ and for each observable, we picked the minimum and maximum values of the $1 \sigma$ bootstrap envelope in the region $T \pm 2 \%$. 


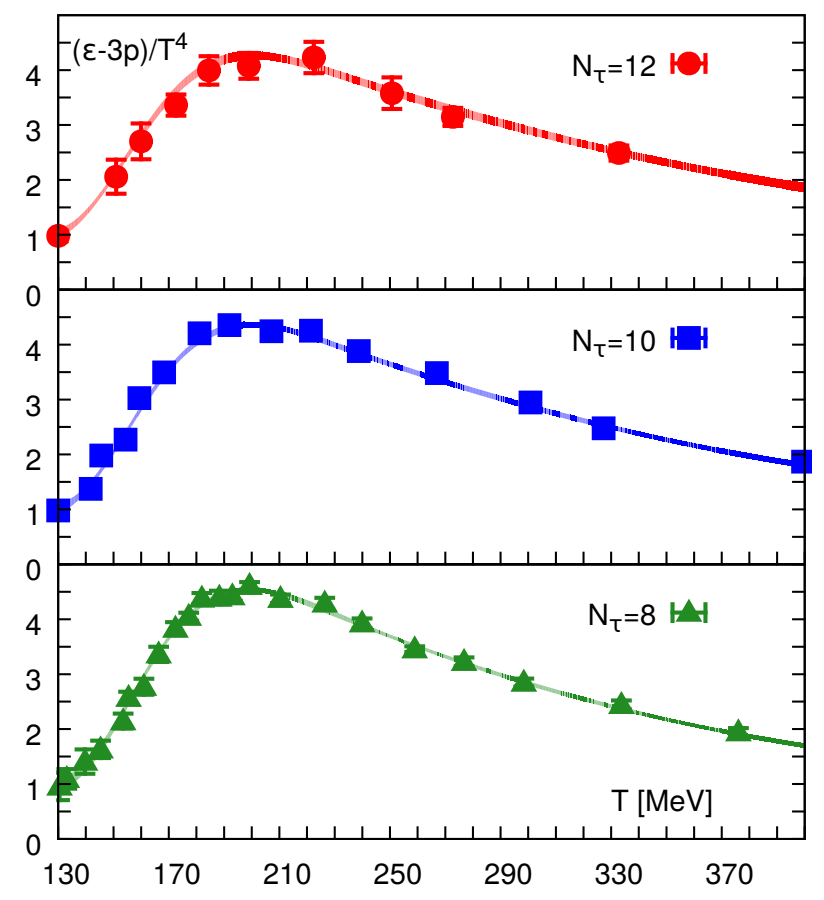

Figure 4: The data for the trace anomaly and the result (thick lines showing the $1 \sigma$ bootstrap error bands) of applying Eq. (14) with $N_{\tau}=8,10$, and 12. The parameters in Eq. (14) and their errors, defining this final fit, were determined from these data as discussed in the text. The error bands shown are generated by the same bootstrap process used to estimate the fit parameters and their errors. The additional $2 \%$ error that is added to the final continuum result to account for the uncertainty in the determination of the temperature scale as discussed in the text is not included in these plots.

This new envelope is then used as the final uncertainty band for all the continuum results shown in the figures and discussed below.

Our continuum extrapolated results for the trace anomaly and other thermodynamic observables are shown in Fig. 5 and the data are given in Table I. For $T<150 \mathrm{MeV}$, the trace anomaly is well approximated by the HRG estimate shown by the solid line in Fig. 5 (left). For $T>150 \mathrm{MeV}$, the $N_{\tau} \geq 8$ lattice results are systematically higher than the HRG estimate as shown in Fig. 3, and the slopes of the HRG and continuum extrapolated curves start to differ as shown in Fig. 5. In the peak region, $(\epsilon-3 p) / T^{4}$ has a maximum of about $4.05(15)$ at $T \sim 204 \mathrm{MeV}$. This maximal value from simulations with the HISQ/tree action is significantly smaller than our previous results with the $\mathrm{p} 4$ and asqtad actions which were incorporated in the HotQCD parametrization [23] of the EoS, as well as in the s95p parametrization of the EoS that is frequently used in hydrodynamic models [45].

The final continuum extrapolated estimates of the pressure, energy density and entropy density are shown in Fig. 5 (right) and compared with HRG predictions for $T<170 \mathrm{MeV}$. Again, there is reasonable agreement for
$T<150 \mathrm{MeV}$. Above $T=150 \mathrm{MeV}$, HRG estimates lie along the lower edge of the error-band of the lattice estimates.

We can now compare our results with the results obtained by the Wuppertal-Budapest Collaboration using the stout action [26]. This comparison is shown in Fig. 6 for the trace anomaly, the pressure and the entropy density. We find good agreement in the trace anomaly with the stout results over the full temperature range $(130-400)$ MeV. Note, however, that above the peak the central values with the stout action lie systematically below ours. As a result, our estimates of the pressure become systematically larger for $T>200 \mathrm{MeV}$. By $T=400 \mathrm{MeV}$, the difference between the central values in the two calculations increases to about $6 \%$. The two results, however, still agree within errors. The difference in the entropy density reaches about $7 \%$ by $T=400 \mathrm{MeV}$, and in this case the two estimates differ by about $2 \sigma$. These differences suggest that more detailed calculations of the trace anomaly at higher temperatures are needed. In particular, it would be important to see if the differences persist at higher temperatures where a comparison with resummed perturbative calculations should be possible (see Sec. V.C).

\section{A. Parametrization of the equation of state}

We close this section by providing an analytical parametrization of the pressure of $(2+1)$-flavor QCD, summarized in Table I, that can be used in phenomenological applications and hydrodynamic modeling of strong interaction matter. We choose an ansatz that incorporates basic features of the low and high temperature limits, i.e., it ensures that the pressure becomes exponentially small at low temperatures and approaches the ideal gas limit at high temperatures. We find that the following parametrization provides an excellent description of all bulk thermodynamic observables discussed in the previous sections, including the specific heat and speed of sound that require second derivatives of $p / T^{4}$ with respect to the temperature to be discussed in the next section,

$$
\begin{gathered}
\frac{p}{T^{4}}=\frac{1}{2}\left(1+\tanh \left(c_{t}\left(\bar{t}-t_{0}\right)\right)\right) . \\
\frac{p_{i d}+a_{n} / \bar{t}+b_{n} / \bar{t}^{2}+c_{n} / \bar{t}^{3}+d_{n} / \bar{t}^{4}}{1+a_{d} / \bar{t}+b_{d} / \bar{t}^{2}+c_{d} / \bar{t}^{3}+d_{d} / \bar{t}^{4}},
\end{gathered}
$$

where $\bar{t}=T / T_{c}$ and the QCD transition temperature $T_{c}=154 \mathrm{MeV}$ is a conveniently chosen normalization. In this parametrization, $p_{i d}=95 \pi^{2} / 180$ is the ideal gas value of $p / T^{4}$ for massless 3 -flavor QCD. It is also the appropriate infinite temperature limiting value for QCD with light and strange quarks that could be refined to include additional perturbative corrections. However, at present we do not see any need for this. We also note that fixing $c_{n}=c_{d}=0$ gives an excellent parametrization of 

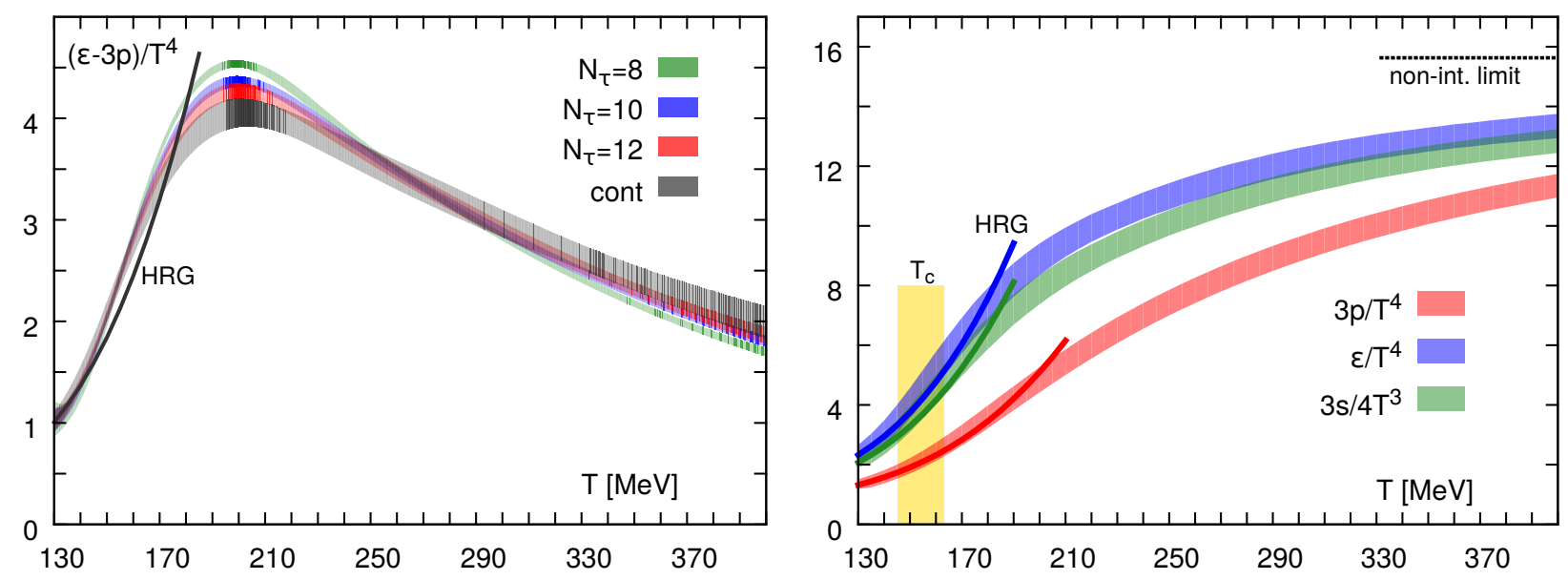

Figure 5: Spline fits to the trace anomaly for several values of the lattice spacing $a T=1 / N_{\tau}$ and the result of our continuum extrapolation (left). Note that the error bands shown here do not include the $2 \%$ scale error. The right hand panel shows suitably normalized pressure, energy density, and entropy density as a function of the temperature. In this case the $2 \%$ scale error is included in the error bands. The dark lines show the prediction of the HRG model. The horizontal line at $95 \pi^{2} / 60$ in the right panel corresponds to the ideal gas limit for the energy density and the vertical band marks the crossover region, $T_{c}=(154 \pm 9) \mathrm{MeV}$.

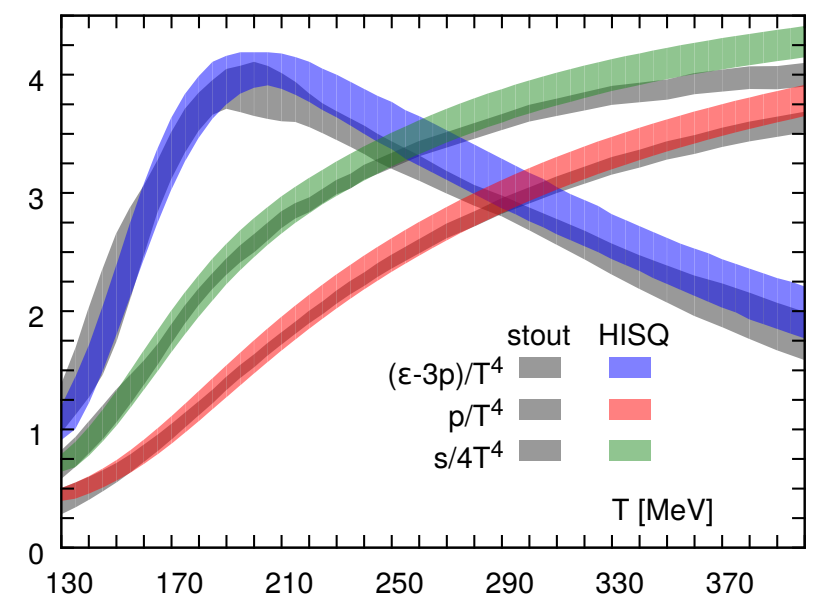

Figure 6: The comparison of the HISQ/tree and stout results for the trace anomaly, the pressure, and the entropy density.

all our numerical data and is in good agreement with the HRG estimate, at least down to $T=100 \mathrm{MeV}$. Furthermore, this parametrization agrees with the $N_{\tau}=8$ data well beyond $T=400 \mathrm{MeV}$.

The values of the parameters in our ansatz for the pressure, Eq. (16), are summarized in Table II. The results of this ansatz for the speed of sound, energy density, and specific heat are compared with our continuum extrapolated error bands in Figs. 7 and 8.

\section{SPECIFIC HEAT, THE SPEED OF SOUND AND DECONFINEMENT}

All thermodynamic quantities, for fixed light and strange quark masses, depend on a single parameterthe temperature. In Section IV, we derived the basic thermodynamic observables $(\epsilon, p, s)$ from the continuum extrapolated trace anomaly $\Theta^{\mu \mu}(T)$. We now discuss two closely related observables that involve second order derivatives of the QCD partition function with respect to the temperature, i.e., the specific heat,

$$
C_{V}=\left.\frac{\partial \epsilon}{\partial T}\right|_{V} \equiv\left(4 \frac{\epsilon}{T^{4}}+\left.T \frac{\partial\left(\epsilon / T^{4}\right)}{\partial T}\right|_{V}\right) T^{3}
$$

and the speed of sound,

$$
c_{s}^{2}=\frac{\partial p}{\partial \epsilon}=\frac{\partial p / \partial T}{\partial \epsilon / \partial T}=\frac{s}{C_{V}} .
$$

The quantity $T \mathrm{~d}\left(\epsilon / T^{4}\right) / \mathrm{d} T$ can be calculated directly from the trace anomaly and its derivative with respect to temperature,

$$
T \frac{\mathrm{d} \epsilon / T^{4}}{\mathrm{~d} T}=3 \frac{\Theta^{\mu \mu}}{T^{4}}+T \frac{\mathrm{d} \Theta^{\mu \mu} / T^{4}}{\mathrm{~d} T} .
$$

These identities show that the estimates for the specific heat and the speed of sound should be of a quality similar to $\epsilon / T^{4}$ or $p / T^{4}$. In Figs. 7 and 8 , we show the agreement between the bootstrap error bands for these quantities and the estimates obtained by taking second order derivatives of the analytic parameterization for $p / T^{4}$ given in Eq. 16. The latter are shown as dark lines inside the bootstrap error bands. 


\begin{tabular}{|c|c|c|c|c|c|c|}
\hline$T[\mathrm{MeV}]$ & $\Theta^{\mu \mu} / T^{4}$ & $p / T^{4}$ & $\epsilon / T^{4}$ & $s / T^{3}$ & $C_{V} / T^{3}$ & $c_{s}^{2}$ \\
\hline 130 & $1.01(-10)(+19)$ & $0.439(-44)(+65)$ & $2.33(-16)(+33)$ & $2.77(-20)(+39)$ & $16.5(-0.9)(+3.0)$ & $0.168(-15)(+6)$ \\
\hline 135 & $1.21(-21)(+23)$ & $0.481(-67)(+69)$ & $2.65(-35)(+38)$ & $3.13(-41)(+44)$ & $20.4(-3.0)(+3.1)$ & $0.153(-10)(+12)$ \\
\hline 140 & $1.46(-24)(+25)$ & $0.529(-72)(+75)$ & $3.05(-41)(+43)$ & $3.58(-47)(+50)$ & $24.5(-3.2)(+3.4)$ & $0.146(-8)(+9)$ \\
\hline 145 & $1.76(-27)(+28)$ & $0.586(-78)(+82)$ & $3.52(-46)(+48)$ & $4.11(-52)(+55)$ & $28.6(-3.4)(+3.6)$ & $0.144(-6)(+7)$ \\
\hline 150 & $2.09(-29)(+30)$ & $0.651(-85)(+89)$ & $4.05(-50)(+52)$ & $4.70(-58)(+60)$ & $32.6(-3.6)(+3.6)$ & $0.144(-6)(+7)$ \\
\hline 155 & $2.43(-31)(+32)$ & $0.726(-93)(+97)$ & $4.61(-54)(+56)$ & $5.34(-62)(+65)$ & $36.2(-3.6)(+3.5)$ & $0.148(-7)(+7)$ \\
\hline 160 & $2.76(-32)(+32)$ & $0.808(-100)(+105)$ & $5.19(-57)(+59)$ & $6.00(-66)(+68)$ & $39.3(-3.4)(+3.3)$ & $0.153(-8)(+9)$ \\
\hline 165 & $3.07(-32)(+31)$ & $0.898(-108)(+112)$ & $5.76(-59)(+60)$ & $6.66(-69)(+70)$ & $41.8(-3.2)(+3.0)$ & $0.159(-9)(+10)$ \\
\hline 170 & $3.34(-31)(+30)$ & $0.994(-115)(+118)$ & $6.32(-60)(+60)$ & $7.32(-70)(+71)$ & $43.8(-2.9)(+2.7)$ & $0.167(-10)(+10)$ \\
\hline 175 & $3.56(-29)(+28)$ & $1.094(-121)(+124)$ & $6.85(-60)(+60)$ & $7.94(-71)(+71)$ & $45.2(-2.6)(+2.4)$ & $0.176(-11)(+10)$ \\
\hline 180 & $3.74(-27)(+25)$ & $1.197(-126)(+129)$ & $7.33(-59)(+59)$ & $8.53(-71)(+70)$ & $46.2(-2.4)(+2.1)$ & $0.185(-11)(+10)$ \\
\hline 185 & $3.88(-25)(+23)$ & $1.302(-130)(+133)$ & $7.78(-58)(+57)$ & $9.08(-71)(+71)$ & $47.0(-2.2)(+1.9)$ & $0.194(-11)(+10)$ \\
\hline 190 & $3.97(-22)(+19)$ & $1.406(-134)(+136)$ & $8.19(-57)(+56)$ & $9.60(-69)(+68)$ & $47.5(-1.9)(+1.7)$ & $0.202(-10)(+10)$ \\
\hline 195 & $4.03(-19)(+16)$ & $1.510(-137)(+139)$ & $8.56(-56)(+54)$ & $10.07(-68)(+67)$ & $47.9(-1.7)(+1.6)$ & $0.210(-10)(+10)$ \\
\hline 200 & $4.05(-16)(+14)$ & $1.613(-140)(+141)$ & $8.89(-54)(+52)$ & $10.50(-67)(+65)$ & $48.1(-1.6)(+1.5)$ & $0.218(-10)(+10)$ \\
\hline 205 & $4.05(-14)(+14)$ & $1.713(-142)(+143)$ & $9.19(-52)(+50)$ & $10.90(-65)(+63)$ & $48.4(-1.5)(+1.6)$ & $0.225(-10)(+10)$ \\
\hline 210 & $4.03(-15)(+15)$ & $1.810(-143)(+143)$ & $9.46(-50)(+48)$ & $11.27(-64)(+62)$ & $48.6(-1.6)(+1.6)$ & $0.232(-10)(+10)$ \\
\hline 215 & $3.99(-16)(+16)$ & $1.904(-144)(+144)$ & $9.70(-48)(+47)$ & $11.61(-62)(+60)$ & $48.8(-1.6)(+1.7)$ & $0.238(-10)(+9)$ \\
\hline 220 & $3.94(-17)(+17)$ & $1.995(-144)(+144)$ & $9.93(-47)(+46)$ & $11.92(-61)(+59)$ & $49.1(-1.7)(+1.8)$ & $0.243(-9)(+9)$ \\
\hline 225 & $3.88(-18)(+17)$ & $2.083(-145)(+144)$ & $10.13(-46)(+45)$ & $12.21(-59)(+58)$ & $49.4(-1.8)(+1.8)$ & $0.247(-9)(+8)$ \\
\hline 230 & $3.82(-18)(+18)$ & $2.168(-145)(+144)$ & $10.32(-45)(+44)$ & $12.49(-59)(+58)$ & $49.8(-1.8)(+1.9)$ & $0.251(-8)(+8)$ \\
\hline 235 & $3.76(-19)(+18)$ & $2.249(-144)(+143)$ & $10.50(-45)(+44)$ & $12.75(-59)(+58)$ & $50.3(-1.9)(+1.9)$ & $0.254(-8)(+7)$ \\
\hline 240 & $3.69(-19)(+19)$ & $2.328(-144)(+143)$ & $10.68(-44)(+44)$ & $13.00(-58)(+58)$ & $50.7(-1.9)(+1.9)$ & $0.256(-8)(+7)$ \\
\hline 245 & $3.63(-20)(+19)$ & $2.403(-144)(+143)$ & $10.84(-44)(+44)$ & $13.24(-58)(+57)$ & $51.1(-1.9)(+1.9)$ & $0.259(-7)(+7)$ \\
\hline 250 & $3.57(-20)(+20)$ & $2.476(-143)(+142)$ & $10.99(-44)(+44)$ & $13.47(-57)(+57)$ & $51.5(-1.9)(+1.9)$ & $0.261(-7)(+6)$ \\
\hline 255 & $3.50(-21)(+20)$ & $2.546(-143)(+142)$ & $11.14(-44)(+44)$ & $13.68(-57)(+57)$ & $51.9(-1.9)(+1.9)$ & $0.264(-7)(+6)$ \\
\hline 260 & $3.44(-21)(+21)$ & $2.613(-143)(+142)$ & $11.28(-44)(+44)$ & $13.89(-58)(+57)$ & $52.2(-1.9)(+1.9)$ & $0.266(-7)(+6)$ \\
\hline 265 & $3.38(-21)(+21)$ & $2.678(-142)(+141)$ & $11.41(-44)(+44)$ & $14.09(-58)(+57)$ & $52.5(-1.9)(+1.8)$ & $0.268(-6)(+6)$ \\
\hline 270 & $3.32(-21)(+21)$ & $2.741(-142)(+141)$ & $11.54(-44)(+44)$ & $14.28(-57)(+57)$ & $52.8(-1.8)(+1.8)$ & $0.270(-6)(+6)$ \\
\hline 275 & $3.26(-21)(+21)$ & $2.801(-141)(+141)$ & $11.66(-44)(+44)$ & $14.46(-57)(+57)$ & $53.1(-1.8)(+1.8)$ & $0.272(-6)(+5)$ \\
\hline 280 & $3.20(-21)(+21)$ & $2.859(-141)(+140)$ & $11.77(-44)(+43)$ & $14.63(-57)(+57)$ & $53.3(-1.8)(+1.7)$ & $0.274(-6)(+5)$ \\
\hline 285 & $3.14(-21)(+21)$ & $2.915(-141)(+140)$ & $11.88(-43)(+43)$ & $14.80(-57)(+57)$ & $53.6(-1.8)(+1.7)$ & $0.276(-5)(+5)$ \\
\hline 290 & $3.08(-21)(+21)$ & $2.969(-140)(+140)$ & $11.99(-43)(+43)$ & $14.95(-57)(+56)$ & $53.8(-1.7)(+1.7)$ & $0.278(-5)(+5)$ \\
\hline 295 & $3.02(-20)(+21)$ & $3.021(-140)(+140)$ & $12.08(-43)(+43)$ & $15.11(-56)(+56)$ & $54.0(-1.7)(+1.7)$ & $0.280(-5)(+5)$ \\
\hline 300 & $2.96(-20)(+21)$ & $3.072(-140)(+139)$ & $12.18(-43)(+43)$ & $15.25(-56)(+56)$ & $54.2(-1.7)(+1.7)$ & $0.282(-5)(+6)$ \\
\hline
\end{tabular}

Table I: Continuum extrapolated results for the trace anomaly $\Theta^{\mu \mu}$, pressure $p$, energy density $\epsilon$, entropy density $s$, specific heat $C_{V}$, and the square of the speed of sound $c_{s}^{2}$ in appropriate units of the temperature $T$. The asymmetry in the errors, given in the two brackets, arises from the $2 \%$ systematic error in asigned to the temperature scale.

\section{A. Speed of sound, the softest point of the EoS and the critical energy density}

In Fig. 7 (top), we show the speed of sound as a function of the temperature and compare our results with those obtained by using the stout action [26]. We find that the HISQ/tree and the stout results agree within the estimated errors. The softest point of the EoS [51] at $T \simeq(145-150) \mathrm{MeV}$, i.e., at the minimum of the speed of sound, lies on the low temperature side of the crossover region. At this point, the speed of sound is only slightly below the corresponding HRG value. This follows from the good agreement between HRG estimates and our lattice QCD results for the energy density and the pressure. Furthermore, the value $c_{s}^{2} \simeq 0.15$ is roughly half way between zero, the value expected at a second order phase transition with diverging specific heat ${ }^{3}$, and the value for an ideal massless gas, $c_{s}^{2}=1 / 3$. At the high temperature end, $T \sim 350 \mathrm{MeV}$, it reaches within $10 \%$ of the ideal gas

\footnotetext{
${ }^{3}$ In the case of QCD the specific heat and therefore also the speed of sound stays finite even at a second order phase transition in the chiral limit.
} 


\begin{tabular}{|c|c|c|c|c|c|c|}
\hline$T[\mathrm{MeV}]$ & $\Theta^{\mu \mu} / T^{4}$ & $p / T^{4}$ & $\epsilon / T^{4}$ & $s / T^{3}$ & $C_{V} / T^{3}$ & $c_{s}^{2}$ \\
\hline 305 & $2.91(-20)(+21)$ & $3.120(-139)(+139)$ & $12.27(-43)(+42)$ & $15.39(-56)(+55)$ & $54.3(-1.7)(+1.7)$ & $0.283(-5)(+6)$ \\
310 & $2.85(-20)(+20)$ & $3.167(-139)(+139)$ & $12.35(-42)(+42)$ & $15.52(-56)(+55)$ & $54.5(-1.7)(+1.7)$ & $0.285(-6)(+6)$ \\
315 & $2.79(-19)(+20)$ & $3.212(-139)(+138)$ & $12.43(-42)(+42)$ & $15.64(-56)(+55)$ & $54.6(-1.7)(+1.7)$ & $0.286(-6)(+6)$ \\
320 & $2.74(-19)(+20)$ & $3.256(-139)(+138)$ & $12.51(-42)(+41)$ & $15.76(-55)(+55)$ & $54.8(-1.7)(+1.7)$ & $0.288(-6)(+6)$ \\
325 & $2.69(-19)(+20)$ & $3.298(-138)(+138)$ & $12.58(-42)(+41)$ & $15.88(-55)(+54)$ & $54.9(-1.7)(+1.7)$ & $0.289(-6)(+7)$ \\
330 & $2.63(-19)(+19)$ & $3.338(-138)(+137)$ & $12.65(-41)(+41)$ & $15.99(-54)(+54)$ & $55.0(-1.7)(+1.7)$ & $0.291(-6)(+7)$ \\
335 & $2.58(-19)(+19)$ & $3.377(-138)(+137)$ & $12.71(-41)(+41)$ & $16.09(-54)(+54)$ & $55.1(-1.7)(+1.8)$ & $0.292(-6)(+7)$ \\
340 & $2.53(-19)(+19)$ & $3.415(-137)(+137)$ & $12.78(-41)(+40)$ & $16.19(-54)(+53)$ & $55.2(-1.7)(+1.8)$ & $0.293(-7)(+7)$ \\
345 & $2.48(-20)(+19)$ & $3.452(-137)(+136)$ & $12.83(-41)(+40)$ & $16.29(-54)(+53)$ & $55.3(-1.7)(+1.8)$ & $0.294(-7)(+7)$ \\
350 & $2.43(-20)(+19)$ & $3.487(-136)(+136)$ & $12.89(-40)(+40)$ & $16.38(-53)(+53)$ & $55.4(-1.8)(+1.9)$ & $0.296(-7)(+7)$ \\
355 & $2.38(-20)(+19)$ & $3.521(-136)(+135)$ & $12.94(-40)(+40)$ & $16.47(-53)(+53)$ & $55.5(-1.8)(+1.9)$ & $0.297(-7)(+7)$ \\
360 & $2.33(-20)(+20)$ & $3.554(-136)(+135)$ & $13.00(-40)(+40)$ & $16.55(-53)(+53)$ & $55.6(-1.8)(+1.9)$ & $0.298(-7)(+7)$ \\
365 & $2.29(-21)(+20)$ & $3.586(-135)(+134)$ & $13.04(-40)(+40)$ & $16.63(-53)(+53)$ & $55.7(-1.9)(+1.9)$ & $0.299(-7)(+7)$ \\
370 & $2.24(-21)(+20)$ & $3.617(-135)(+134)$ & $13.09(-40)(+40)$ & $16.71(-53)(+53)$ & $55.8(-1.9)(+2.0)$ & $0.300(-7)(+7)$ \\
375 & $2.20(-21)(+20)$ & $3.647(-134)(+134)$ & $13.14(-40)(+40)$ & $16.78(-53)(+53)$ & $55.8(-1.9)(+2.0)$ & $0.301(-7)(+7)$ \\
380 & $2.15(-22)(+21)$ & $3.675(-134)(+133)$ & $13.18(-40)(+40)$ & $16.85(-53)(+53)$ & $55.9(-2.0)(+2.0)$ & $0.302(-7)(+7)$ \\
385 & $2.11(-22)(+21)$ & $3.703(-134)(+133)$ & $13.22(-40)(+41)$ & $16.92(-53)(+53)$ & $56.0(-2.0)(+2.0)$ & $0.302(-7)(+7)$ \\
390 & $2.07(-22)(+21)$ & $3.730(-133)(+132)$ & $13.26(-40)(+41)$ & $16.99(-53)(+53)$ & $56.1(-2.0)(+2.1)$ & $0.303(-7)(+7)$ \\
395 & $2.03(-22)(+22)$ & $3.756(-133)(+132)$ & $13.30(-40)(+41)$ & $17.05(-53)(+53)$ & $56.2(-2.0)(+2.1)$ & $0.304(-7)(+7)$ \\
400 & $1.99(-22)(+22)$ & $3.782(-132)(+132)$ & $13.34(-40)(+41)$ & $17.12(-53)(+53)$ & $56.2(-2.1)(+2.1)$ & $0.304(-7)(+7)$ \\
\hline
\end{tabular}

Table I continued

\begin{tabular}{|c|c|c|c|c|}
\hline$c_{t}$ & $a_{n}$ & $b_{n}$ & $c_{n}$ & $d_{n}$ \\
3.8706 & -8.7704 & 3.9200 & 0 & 0.3419 \\
\hline \hline$t_{0}$ & $a_{d}$ & $b_{d}$ & $c_{d}$ & $d_{d}$ \\
0.9761 & -1.2600 & 0.8425 & 0 & -0.0475 \\
\hline
\end{tabular}

Table II: Parameters used in the ansatz given in Eq. (16) for the pressure of $(2+1)$-flavor QCD in the temperature interval $T \in[100 \mathrm{MeV}, 400 \mathrm{MeV}]$.

value.

The softest point of the EoS is of interest in the phenomenology of heavy ion collisions as it characterizes the temperature and energy density range in which the expansion and cooling of matter slows down. The system spends a longer time in this temperature range, and one expects to observe characteristic signatures from this regime. To facilitate a more direct comparison with experiments, we show $c_{s}^{2}$ as a function of the energy density in physical units in Fig. 7 (bottom) using the parametrization given in Eq. 16 to convert temperature to energy density. At the softest point, the energy density is only slightly above that of normal nuclear matter, $\epsilon_{\text {nuclear }}=150 \mathrm{MeV} / \mathrm{fm}^{3}$. In the crossover region, $T_{c}=(154 \pm 9) \mathrm{MeV}[5]$, the energy density varies from $180 \mathrm{MeV} / \mathrm{fm}^{3}$ at the lower edge to $500 \mathrm{MeV} / \mathrm{fm}^{3}$ at the upper edge, slightly above the energy density inside the proton $\epsilon_{\text {proton }}=450 \mathrm{MeV} / \mathrm{fm}^{3}$.

The QCD crossover region, thus, starts at or close to the softest point of the EoS and the entire crossover region corresponds to relatively small values of the energy density, $(1.2-3.1) \epsilon_{\text {nuclear }}$. This value is about a factor of four smaller than that of an ideal quark-gluon gas in this temperature range. In the next subsection, we will discuss to what extent this has consequences for the size of fluctuations in the energy density, i.e., the specific heat.

\section{B. Specific heat and deconfinement}

The intuitive characterization of deconfinement at the QCD phase transition is that the liberation of many new degrees of freedom give rise to a rapid increase in the energy density, ideally with an infinite slope at $T_{c}$ as in a conventional second order phase transition. This rapid rise would then show up as a peak (or even a divergence) in the specific heat, which could serve as an indicator for the pseudo-critical (or critical) temperature. However, the specific heat of $(2+1)$-flavor QCD, shown in Fig. 8, exhibits a rapid increase but no peak. In the crossover region, $C_{V} / \epsilon \simeq 8 / T$ is a factor of two larger than for an ideal quark-gluon gas; the specific heat reaches about half of its ideal gas value, $\left(C_{V} / T^{3}\right)_{\text {ideal }}=4\left(\epsilon / T^{4}\right)_{\text {ideal }}=$ $95 \pi^{2} / 15$; and the energy density reaches only about one quarter of its limiting high temperature, ideal gas value.

The analysis of the quark-mass dependence of the QCD transition temperature, the chiral condensate and, in particular, the peak in the chiral susceptibility suggest that for physical values of the quark masses QCD is sufficiently close to the chiral limit to be sensitive to the chiral phase transition [5] and exhibit an almost universal, pseudo-critical behavior controlled by it. The peak observed in the chiral susceptibility is dominated by the sec- 

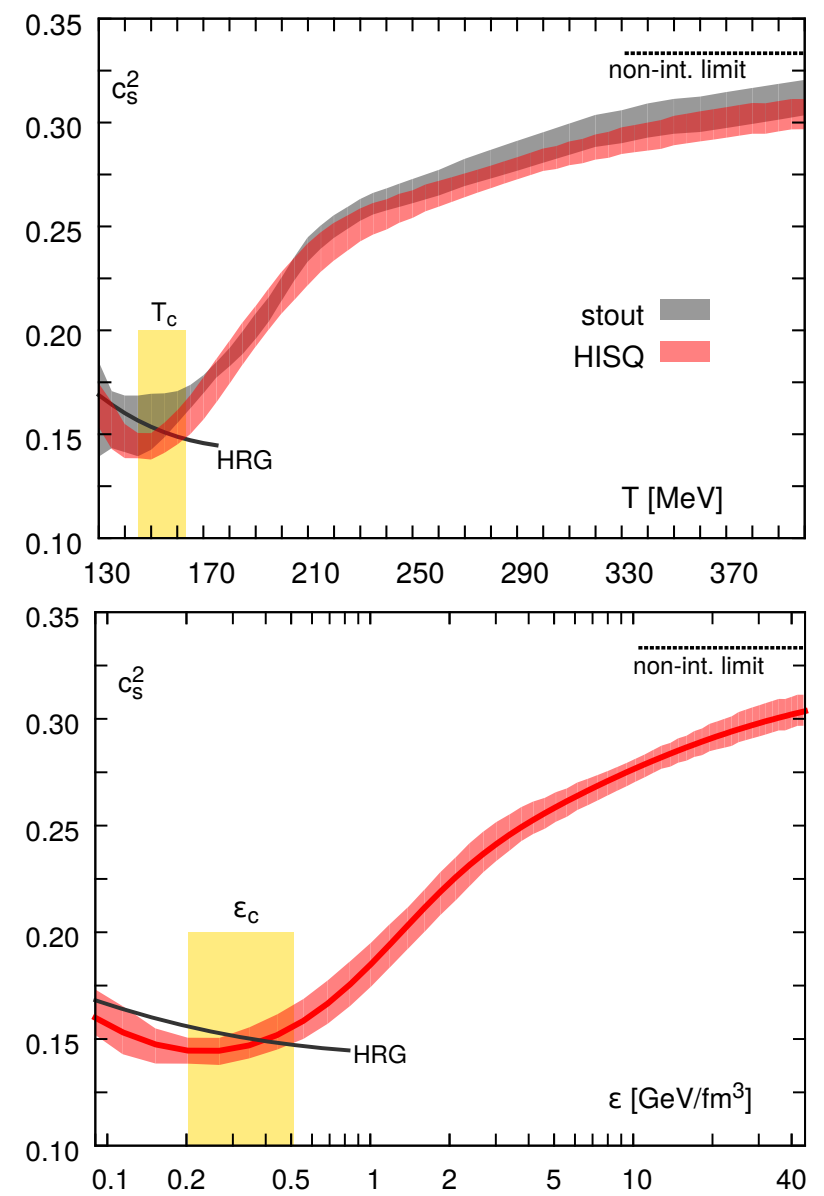

Figure 7: The speed of sound squared from lattice QCD and the HRG model versus temperature (top) and energy density (bottom). In the upper figure, our results (HISQ) are compared with those obtained with the stout action [26]. The vertical band marks the location of the crossover region $T_{c}=(154 \pm 9) \mathrm{MeV}$ in the upper figure and the corresponding range in energy density,$\epsilon_{c}=(0.18-0.5) \mathrm{GeV} / \mathrm{fm}^{3}$, in the lower figure. The dark line within each error band is the prediction of the analytical parameterization given in Eq. (16).

ond derivative of the singular part of the free energy with respect to the quark mass [5]. Extending that generic scaling analysis, one may have expected that, for physical quark masses, the pseudo-critical behavior would also lead to large fluctuations in the energy density and that the specific heat would exhibit a peak in the crossover region controlled by the second derivative with respect to temperature of the same singular part of the free energy.

There may be at least two reasons for the difference in behavior between the chiral susceptibility and the specific heat, which are second derivatives of the partition function with respect to the quark mass and the temperature, respectively. First, thermal fluctuations are controlled by the thermal critical exponent $\alpha$, i.e., $C_{V} / T^{3} \sim\left|T-T_{c}\right|^{-\alpha}$. In the 3-d $O(4)$ universality class, which is relevant for the chiral phase transition, the exponent $\alpha \simeq-0.21$ is negative [52]. Consequently, unlike

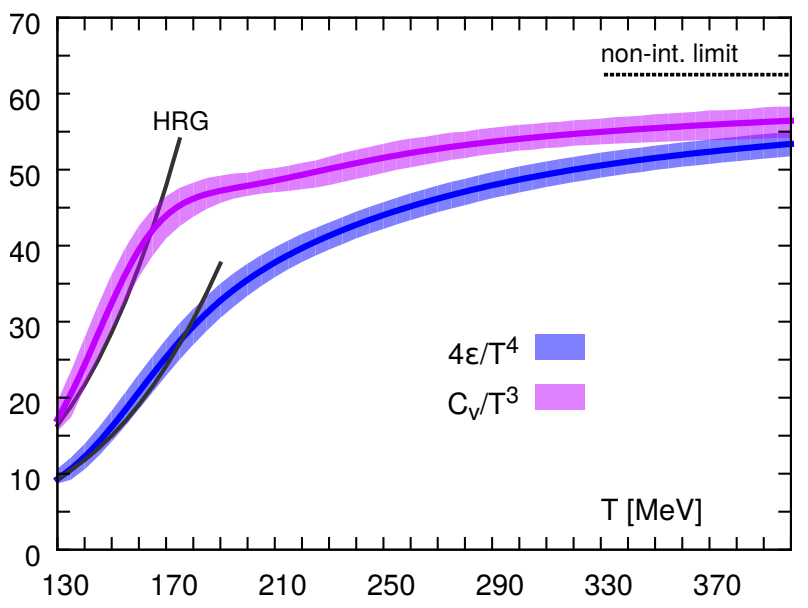

Figure 8: Error bands showing the continuum extrapolation of the specific heat and energy density and solid lines obtained from the parametrization given in Eq. (16). Also shown are the HRG estimates at low temperatures and the ideal gas limit at high temperatures.

the chiral susceptibility, the specific heat stays finite at $T_{c}$ even in the chiral limit. The singular part of the free energy [52], which gives the leading temperature dependence in the vicinity of $T_{c}$, contributes only a cusp in $C_{V}$. This can be seen by examining the energy density near $T_{c}$,

$$
\frac{\epsilon}{T^{4}}=e_{0}+e_{1}\left(\frac{T-T_{c}}{T_{c}}\right)+\mathcal{O}\left(\left|T-T_{c}\right|^{1-\alpha}\right),
$$

where the dominant contribution, $e_{0}$, comes from the regular part and the singular contributions, $\sim\left|T-T_{c}\right|^{1-\alpha}$, are sub-dominant. From Eq. (17), we get

$$
\frac{C_{V}}{T^{3}}=c_{0}+\frac{A^{ \pm}}{\alpha}\left|\frac{T-T_{c}}{T_{c}}\right|^{-\alpha}+\mathcal{O}\left(T-T_{c}\right),
$$

with $c_{0}=4 e_{0}+e_{1}$ and $A^{+}\left(A^{-}\right)$are the amplitudes above (below) $T_{c}$. The ratio of these amplitudes is universal and positive; $A^{+} / A^{-}=1.842(43)$ in the 3 -d O(4) universality class [52]. Since $\alpha$ is negative, the singular part gives only a cusp, which should persist in the chiral limit but may not be easy to detect if the regular contributions are large.

The second reason for the lack of a peak in $C_{V} / T^{3}$ is that the contributions from the regular part of the free energy are large in the high temperature phase [52], and are $\mathcal{O}\left(g^{0}\right)$ at infinite temperature. Furthermore, as discussed above, the regular terms dominate even in the crossover region. To make this observation more explicit, we note from Eq. (17) that $C_{V} / T^{3}$ can be written in terms of the energy density, $\epsilon / T^{4}$, and its derivative,

$$
T \frac{\mathrm{d} \epsilon / T^{4}}{\mathrm{~d} T} \equiv \frac{\bar{C}_{V}}{T^{3}} .
$$

The dominant singular terms are contained in the second term $\left(\bar{C}_{V} / T^{3}\right)$ or, more specifically, in the temperature derivative of the trace anomaly, i.e., the second 


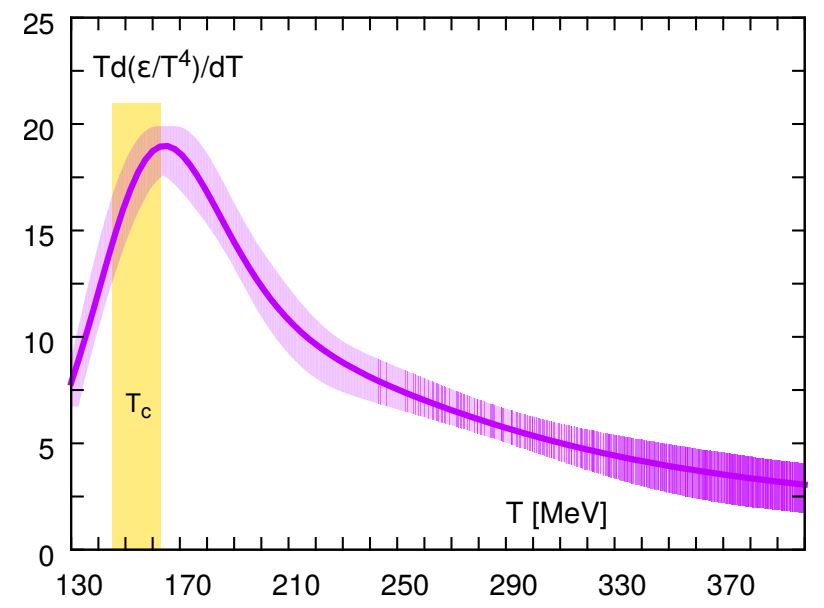

Figure 9: Derivative of $\epsilon / T^{4}$ with respect to temperature. The vertical band gives the chiral crossover temperature determined from the location of the peak in the disconnected chiral susceptibility.

term in Eq. (19). The contribution of the regular terms to $\bar{C}_{V} / T^{3}$ is strongly suppressed at high temperatures; it is zero in the infinite temperature ideal gas limit and receives contributions starting at $\mathcal{O}\left(g^{4}\right)$ in perturbation theory. Thus, while $C_{V}$ and $\bar{C}_{V}$ have identical leading contributions from the singular part near $T_{c}$, the contribution from the regular part is much smaller in $\bar{C}_{V}$. Consequently, the singular behavior is not masked and $\bar{C}_{V}$ has a pronounced peak close to the chiral crossover region as shown in Fig. 9. To summarize, the location of the peak in the temperature derivative of $\epsilon / T^{4}$ is a good indicator of deconfinement, i.e., the liberation of quarkgluon degrees of freedom, and occurs close to the chiral transition in QCD as shown in Fig. 9.

\section{Approach to the perturbative limit}

In this subsection, we discuss how our results for the $(2+1)$-flavor EoS connect to analytic calculations at high temperatures.

At sufficiently high temperatures, thermodynamics should be describable in terms of a weakly interacting quark-gluon gas, and at infinite temperature all thermodynamic quantities will converge to the ideal gas limit. Plots in Fig. 5 show that at our highest temperature value, $T=400 \mathrm{MeV}$, the entropy and energy density and pressure are still 13\%, $18 \%$ and $27 \%$, respectively, below the ideal gas limit. In contrast to other quantities, e.g., susceptibilities of conserved charge fluctuations, these deviations from the ideal gas limit are still quite large. This is probably due to large nonperturbative contributions in the gluonic sector of QCD which are present in bulk thermodynamic observables but are suppressed in observables that, at tree level, only depend on the quark sector of QCD.

Although, for some observables, resummation [53] or dimensional reduction [54] based perturbative calculations show good agreement with lattice QCD calculations already at temperatures $T \sim 400 \mathrm{MeV}$, for others this is not the case. In particular, their functional dependence on temperature is still significantly different in this temperature range, which can lead to larger differences in higher order derivatives between perturbative and lattice QCD calculations. As our current continuumextrapolated EoS is limited to $T<400 \mathrm{MeV}$, we cannot perform a detailed comparison with perturbation theory but point out a few qualitative features.

We have shown in Fig. 6 that continuum-extrapolated results for the trace anomaly obtained with the stout and the HISQ discretization schemes agree within errors. At high temperatures, however, the HISQ results are systematically above the stout results. This propagates into other thermodynamic observables, e.g., the pressure. The systematic differences, however, cancel to a large extent in ratios. For example, the ratio of the trace anomaly and the pressure,

$$
\frac{\Theta^{\mu \mu}}{p}=\frac{\epsilon}{p}-3,
$$

is in excellent agreement between the two calculations and thus provides a good starting point for a comparison with high temperature perturbative calculations. In Fig. 10, we show results for the ratio $\Theta^{\mu \mu} / p$ and compare with perturbative calculations performed in the Hard Thermal Loop (HTL) [53] and Electrostatic QCD (EQCD) [54] schemes. The broad band for the three-loop HTL calculation corresponds to varying the renormalization scale in the interval $\mu=(1-4) \pi T$ and the black line in this band corresponds to $\mu=2 \pi T$. The EQCD and HTL results for $\mu=2 \pi T$ are in good agreement, and the lattice QCD results approach these estimates for $T \gtrsim 500 \mathrm{MeV}$.

In Fig. 11, we compare the three-loop HTL estimates with lattice QCD calculations of the trace anomaly (top) and the pressure (bottom). Also shown, with a dashed line in Figs. 10 and 11, is the result of an $\mathcal{O}\left(g^{6}\right)$ calculation performed in the dimensional reduction scheme (EQCD). The lattice QCD results are in qualitative agreement with these perturbative calculations, with the $\mathcal{O}\left(g^{6}\right)$ EQCD estimate lying below the lattice QCD results for the trace anomaly and above for the pressure at $T=400 \mathrm{MeV}$.

One could try fixing the scale uncertainty in the HTL calculation by matching one of the observables to the lattice QCD result, e.g., the pressure. Results for other observables, e.g., the trace anomaly would then be parameter free predictions. It is clear from Fig. 11 that such a simultaneously agreement between HTL and lattice QCD calculations of $p / T^{4}$ and $(\epsilon-3 p) / T^{4}$ is not forthcoming. Making the HTL and the lattice QCD estimates agree for the trace anomaly by reducing the value for the renormalization scale $\mu$ would decrease the HTL results for $p / T^{4}$ even further and, thereby, increase the deviation from the lattice QCD results. 


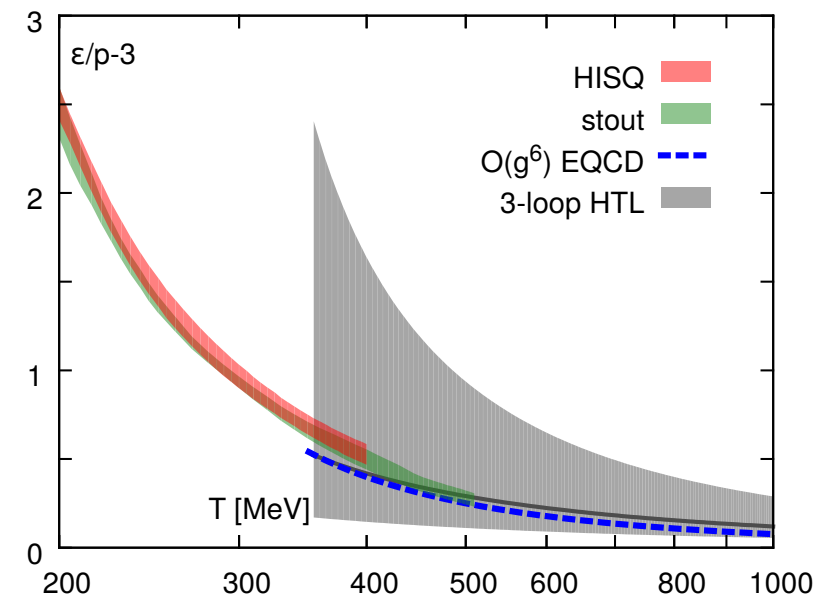

Figure 10: The ratio of the trace anomaly and the pressure from (2+1)-flavor QCD calculations with the HISQ and stout actions, respectively. These results are compared to HTL and EQCD (dashed line) calculations. The black line corresponds to the HTL calculation with renormalization scale $\mu=2 \pi T$.

Lastly, the EQCD result for the pressure in the temperature range $(400-1000) \mathrm{MeV}$ is about $10 \%$ larger than the HTL result with $\mu=2 \pi T$. To resolve the open question whether at these high temperatures the pressure obtained from lattice QCD calculations is better described by the HTL or the EQCD calculations requires lattice simulations at higher temperatures.

\section{CONCLUSIONS}

We have calculated the trace anomaly and the equation of state in $(2+1)$-flavor QCD with almost physical quark masses using the HISQ/tree action on lattices with temporal extent $N_{\tau}=6,8,10$, and 12 . We find that the lattice discretization errors in the HISQ/tree action are small, and we obtain reliable continuum extrapolated results for a number of thermodynamic quantities for $130 \mathrm{MeV}<T<400 \mathrm{MeV}$. In fact, the trace anomaly calculated on the $N_{\tau}=12$ lattices agrees with the continuum-extrapolated results within errors. Our main results are summarized in Figs. 1, 5, and 6. Based on these results, we propose in Eq. 16 an analytical parameterization of the pressure for use in phenomenological studies that matches the HRG estimates below $T=$ $130 \mathrm{MeV}$ and the lattice data between $(130-400) \mathrm{MeV}$.

We have compared our new results obtained using the HISQ/tree action with our previous calculations performed using the asqtad and the p4 actions [23], and with the recent continuum extrapolated stout results [26]. For $T<300 \mathrm{MeV}$, the HISQ/tree results are very different from the results obtained using the $\mathrm{p} 4$ and the asqtad actions on $N_{\tau}=8$ lattices, i.e., without an extrapolation to the continuum limit. At higher temperatures, the results show reasonable agreement as expected since all three actions have small lattice artifacts.

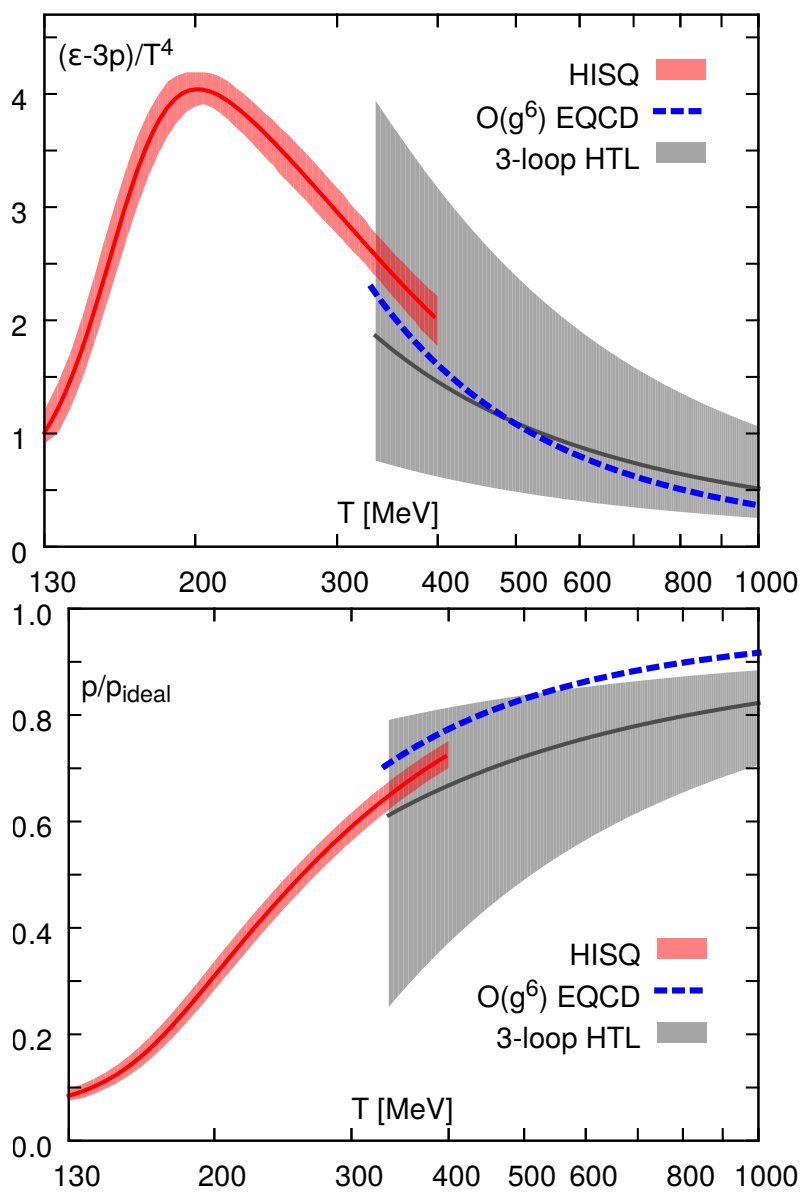

Figure 11: Comparison of the $(2+1)$-flavor calculation of the trace anomaly (top) and pressure (bottom) with HTL and EQCD (dashed line) calculations. The black line corresponds to the HTL calculation with renormalization scale $\mu=2 \pi T$. Note that this solid line would move up for the trace anomaly and move down for the pressure if the scale $\mu$ in HTL is reduced.

Results for our continuum extrapolated trace anomaly presented in Sec. IV agree well with those from the stout action [26]. The discrepancy between the HotQCD results and the stout results discussed in $[23,26]$ was due to the large cutoff effects in the previous estimates with the p4 and the asqtad actions and because our earlier results had not been extrapolated to the continuum limit.

We find reasonably good agreement for the pressure obtained using the stout and the HISQ/tree actions for $T<300 \mathrm{MeV}$ as shown in Fig. 6. At higher temperatures, there is some tension between the two estimates because the results for the trace anomaly, $\Theta^{\mu \mu}(T) / T^{4}$, obtained with the HISQ/tree action lie systematically above those from the stout action. Consequently, the pressure, which is the integral of $\Theta^{\mu \mu}(T) / T^{5}$, will start to differ significantly at high temperatures if the observed trends persist. In this paper, we focused on the temperature region $130 \mathrm{MeV}<T<400 \mathrm{MeV}$, which is the most relevant for phenomenological applications. 
Over this temperature range, the difference is unlikely to have a significant effect on the modeling of the hydrodynamic evolution of the system produced in heavy ion collisions (see the discussion in Ref. [45]). It is important to check, however, if this tension persists at higher temperatures, especially if one wants to determine to what extent the quark-gluon plasma is strongly or weakly coupled by comparing lattice and resummed perturbation theory results for the pressure or for the entropy density. Such calculations are left for future studies.

\section{Acknowledgments}

This work has been supported in part by contracts DE-AC02-98CH10886, DE-AC52-07NA27344, DE-FC0212ER41879, DE-FG02-92ER40699, DE-FG02-91ER40628, DE-FG02-91ER-40661, DE-FG02-04ER-41298, DE-KA-14-01-02, DE-SC0010120 with the U.S. Department of Energy, and NSF grants PHY07-03296, PHY0757333, PHY10-67881, PHY08-57333, PHY-1212389, and PHY13-16748, the Bundesministerium für Bildung und Forschung under grant 06BI9001 and 05P12PBCTA, and the EU Integrated Infrastructure Initiative HadronPhysics3. The numerical simulations have been performed on BlueGene/L computers GPU cluster (Edge) at Lawrence Livermore National Laboratory (LLNL), the New York Center for Computational Sciences (NYCCS) at Brookhaven National Laboratory, on BlueGene/P and BlueGene/Q computers at Argonne Leadership Computing facility, on BlueGene/P computers at NIC, Juelich, US Teragrid (Texas Advanced Computing Center), at NERSC, GPU clusters at University of Bielefeld, the OCuLUS cluster at University of Paderborn, and on clusters of the USQCD collaboration in JLab and FNAL. This research was supported in part by Lilly Endowment, Inc., through its support for the Indiana University Pervasive Technology Institute, and in part by the Indiana METACyt Initiative. The Indiana METACyt Initiative at IU is also supported in part by Lilly Endowment, Inc. We thank Nathan Brown for help with the $w_{0}$ scale calculations and Michael Strickland for providing us with data from the HTL resummed perturbative calculations.

\section{Appendix A: HISQ ensembles and topological charge history}

\section{HISQ ensembles}

To simulate the HISQ/tree action, we use the same Rational Hybrid Monte Carlo algorithm [55] with mass preconditioning [56] as in the previous study Ref. [5]. Details of these simulations are given in Ref. [57] and in Table III we present the key lattice parameters of our simulation, namely the gauge coupling $\beta=10 / g^{2}$, the quark masses, the lattice dimensions, the accumulated statistics in terms of molecular dynamics time units (TU), and the length of the trajectories. The zero temperature lattices were saved every 5 TUs (or 6 TU for the fine lattices), and the finite temperature lattices were saved every 10 TUs.

\section{Topological charge history}

The topological charge history gives an indication of the ergodicity of the molecular dynamics evolution. Ideally, we want a reasonably good coverage of the most probable topological charge sectors. This occurs when tunneling between the topological charge sectors is reasonably frequent. It is expected that the tunneling rate decreases as the lattice spacing is decreased. Therefore, to test ergodicity in our molecular dynamics evolution, we look at the least favorable case, namely our finest lattices.

In our previous study we checked the evolution of the topological charge in our simulations down to lattice spacings $a=0.066 \mathrm{fm}$, and found that it fluctuated quite rapidly [5]. In the present study the lattice spacing for our two finest lattices corresponding to $\beta=7.596$ and $\beta=7.825$ is smaller still, namely, $a=0.049 \mathrm{fm}$ and $0.041 \mathrm{fm}$, respectively. In Fig. 12 we show the evolution of the topological charge for those two ensembles. The figures show a slower tunneling rate than in our previous study, but we still see a reasonable coverage of the topological charge sectors.

\section{Appendix B: Lattice scale}

To translate lattice observables to physical dimensionfull quantities, we need to measure the lattice spacing. We consider three methods for determining the scale: the static-quark-potential parameters $r_{1}$ or $r_{0}$, the gradient flow parameter $w_{0}$ [41], and the kaon decay constant $f_{K}$. Our preferred method uses the static quark potential. The other methods are used as a cross check. We discuss here the the former three ways to set the scale and defer the discussion of $f_{K}$ to Appendix C.

\section{Static quark potential}

The static quark potential is used indirectly to set the scale. In brief, a standard radius $r_{0}$ [38] or $r_{1}$ [39] is calculated from the measured heavy quark potential using Eqs. (1) and (2). On any ensemble, the standard radii are first determined in lattice units: $r_{0} / a$ and $r_{1} / a$. The values of $r_{0}$ and $r_{1}$ in the continuum limit are known in physical units from other lattice studies, based on, for example, the experimental value of $f_{\pi}$. From them and $r_{0} / a$ one can infer the value of $a$.

The static potential for the HISQ/tree action has been studied in Ref. [5] for a large range of gauge couplings $\beta$. We extended these studies in the following 

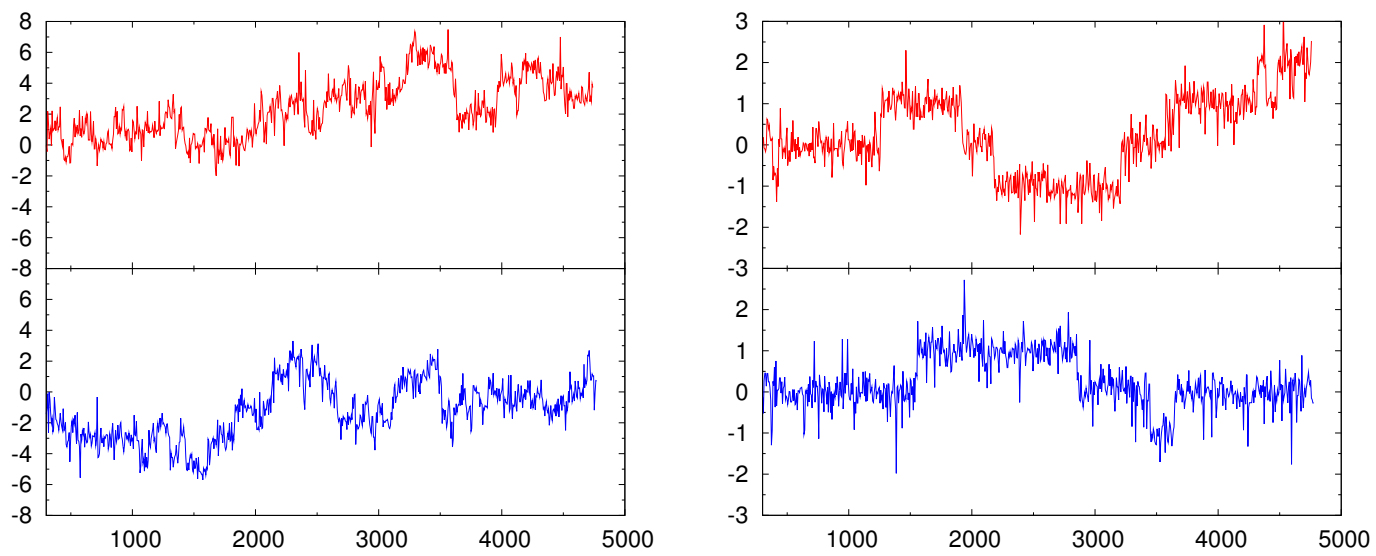

Figure 12: The evolution of topological charge in Monte-Carlo time for $\beta=7.596$ (left) and $\beta=7.825$ (right). The top and bottom panels correspond to two different streams for hybrid Monte-Carlo evolution.

ways. We improved significantly the statistical accuracy of the calculation of the static potential at the highest beta values considered in Ref. [5], namely for $\beta=6.88,6.95,7.03,7.15$ and 7.28 . To them we added calculations of the potential at $\beta=6.740,7.373,7.596$, and 7.825. As in our previous study, we used Coulomb gauge fixing and calculated the potential from the correlation of two Wilson lines of length $\tau$ at distance $R$. The potential is then obtained from the logarithm of the ratio of two such correlators at neighboring $\tau$ values. We fit this ratio to a constant plus a term that decays exponentially in Euclidean time $\tau$ in the interval $\left[\tau_{\min }, \tau_{\max }\right]$. We also studied the variation of the potential due to different choices $\left[\tau_{\min }: \tau_{\max }\right]$ to estimate possible systematic errors. To be specific, we used finally [2:4], [3:7], [4:9], [3:9], [4:9], [4:10], [4:10], [5:8], and [6:11] for $\beta=6.740,6.88,6.95,7.03,7.15,7.28,7.373,7.596$, and 7.825 , respectively.

The scales $r_{0} / a$ or $r_{1} / a$ are then determined by separately fitting the resulting potential to a Coulomb-pluslinear-plus-constant form in the $r$-intervals around the values of $r_{1}$ and $r_{0}$, respectively. We vary the fit intervals, and the variations in the extracted values of $r_{1} / a$ and $r_{0} / a$ are used as estimates of systematic errors. In most cases, the systematic errors are larger than the statistical ones. The statistical and systematic errors are added in quadrature to estimate the total error for $r_{0}$ and $r_{1}$. The values $r_{0} / a, r_{1} / a$ and their ratios $r_{0} / r_{1}$ determined in this study as well as from Ref. [5] are given in Table IV. As in our previous study, the ratio $r_{0} / r_{1}$ appears to be independent of $\beta$ (lattice spacing) within the estimated errors [5]. Accordingly, as before, we fit the values of $r_{0} / r_{1}$ given in Table IV to a constant for $\beta \geq 6.423$ and obtain

$$
\left(r_{0} / r_{1}\right)_{\text {cont }}=1.5092 \pm 0.0039, \chi^{2} / \text { dof }=0.22 .
$$

This value agrees well with our previous estimate $r_{0} / r_{1}=$ $1.508(5)$ [5]. We also fit the ratio $r_{0} / r_{1}$ using only the data for $\beta \geq 6.664$ and $\beta \geq 6.608$, obtaining $r_{0} / r_{1}=$ $1.5083(44)$ and $r_{0} / r_{1}=1.5075(43)$ with similar $\chi^{2} /$ dof. These values agree well with the one given in Eq. (B1). Therefore we use Eq. (B1) as our final estimate for $r_{0} / r_{1}$.

To determine the lattice spacing as function of $\beta$, we fit $a / r_{1}$ to the Allton-type ansatz [58],

$$
\begin{gathered}
\frac{a}{r_{1}}=\frac{c_{0} f(\beta)+c_{2}(10 / \beta) f^{3}(\beta)}{1+d_{2}(10 / \beta) f^{2}(\beta)}, \\
f(\beta)=\left(\frac{10 b_{0}}{\beta}\right)^{-b_{1} /\left(2 b_{0}^{2}\right)} \exp \left(-\beta /\left(20 b_{0}\right)\right) .
\end{gathered}
$$

Here $b_{0}$ and $b_{1}$ are the well-known coefficients of the two-loop beta function, which for the three-flavor case are $b_{0}=9 /\left(16 \pi^{2}\right), b_{1}=1 /\left(4 \pi^{4}\right)$. At small $\beta$, the parameter $r_{1}$ is small in lattice units. Therefore, to avoid possibly large discretization effects, for $\beta=6.423$, where $r_{0} / a$ is more reliably determined, we use $r_{1} / a=$ $r_{0} / a /\left(r_{0} / r_{1}\right)_{\text {cont }}$ with $\left(r_{0} / r_{1}\right)_{\text {cont }}$ from Eq. (B1) (see discussions in Ref. [5]). The fit gives $\chi^{2} /$ dof $=0.25$ and

$$
\begin{gathered}
c_{0}=43.1 \pm 0.3, \\
c_{2}=343236 \pm 41191, \\
d_{2}=5514 \pm 755 .
\end{gathered}
$$

The errors on the above fit parameters have also been estimated using the bootstrap method which gives very similar results. The differences between the above parametrization of $r_{1} / a$ and the previous one from Ref. [5] are less than $0.2 \%$ for $\beta<6.8$. For larger beta values the differences are larger but do not exceed $1.3 \%$. To convert all quantities to physical units, as in Ref. [5], we use the value $r_{1}=0.3106 \mathrm{fm}$ from [40].

To test the uncertainty in the scale parametrization, we also fit the data for $a / r_{1}$ to the asymptotic form $f(\beta)$ times a smoothing spline. The smoothing spline is determined by minimizing the $\chi^{2}$ plus the integral of the 


\begin{tabular}{|c|c|c|c|c|c|c|c|c|c|}
\hline \multirow[b]{2}{*}{$\beta$} & \multirow[b]{2}{*}{$m_{l}$} & \multirow[b]{2}{*}{$m_{s}$} & \multicolumn{3}{|c|}{$T=0$} & \multirow{2}{*}{$\frac{N_{\tau}=6}{T U}$} & \multirow{2}{*}{$\frac{N_{\tau}=8}{T U}$} & \multirow{2}{*}{$\frac{N_{\tau}=10}{T U}$} & \multirow{2}{*}{$\frac{N_{\tau}=12}{T U}$} \\
\hline & & & $N_{s}^{3} \times N_{\tau}$ & $T U$ & length & & & & \\
\hline 5.900 & 0.00660 & 0.1320 & $24^{3} \times 32$ & 3700 & $1 / 4$ & 30290 & - & - & - \\
\hline 5.950 & 0.00615 & 0.1230 & $24^{3} \times 32$ & 4715 & $1 / 4$ & 30990 & - & & - \\
\hline 6.000 & 0.00569 & 0.1138 & $24^{3} \times 32$ & 4890 & $1 / 3$ & 31730 & - & - & - \\
\hline 6.025 & 0.00550 & 0.1100 & $24^{3} \times 32$ & 5250 & $1 / 3$ & 33990 & - & - & - \\
\hline 6.050 & 0.00532 & 0.1064 & $24^{3} \times 32$ & 4655 & $1 / 3$ & 32100 & 74210 & - & - \\
\hline 6.075 & 0.00518 & 0.1036 & $24^{3} \times 32$ & 4085 & $1 / 3$ & 32990 & - & - & - \\
\hline 6.100 & 0.00499 & 0.0998 & $28^{3} \times 32$ & 4190 & $1 / 3$ & 39900 & - & - & - \\
\hline 6.125 & 0.00483 & 0.0966 & $32^{4}$ & 8645 & $1 / 3$ & 32990 & 67720 & - & - \\
\hline 6.150 & 0.00468 & 0.0936 & $24^{3} \times 32$ & 7795 & $1 / 3$ & 31130 & - & - & - \\
\hline 6.175 & 0.00453 & 0.0906 & $32^{4}$ & 9080 & $1 / 3$ & 30990 & 60480 & - & - \\
\hline 6.195 & 0.00440 & 0.0880 & $32^{4}$ & 8445 & $1 / 2$ & 33150 & 25790 & - & - \\
\hline 6.245 & 0.00415 & 0.0830 & $32^{4}$ & 8505 & $1 / 2$ & 30990 & 28070 & - & - \\
\hline 6.285 & 0.00395 & 0.0790 & $32^{4}$ & 7350 & $1 / 2$ & 30990 & 40250 & - & - \\
\hline 6.341 & 0.00370 & 0.0740 & $32^{4}$ & 6705 & 1 & 30990 & 33310 & - & - \\
\hline 6.354 & 0.00364 & 0.0728 & $32^{4}$ & 8000 & 1 & 30990 & 220312 & - & - \\
\hline 6.390 & 0.00347 & 0.0694 & $32^{4}$ & 4602 & 1 & - & 269636 & - & - \\
\hline 6.423 & 0.00335 & 0.0670 & $32^{4}$ & 7970 & 1 & 30990 & 113315 & - & - \\
\hline 6.460 & 0.00320 & 0.0640 & $32^{3} \times 64$ & 2900 & 1 & - & 84841 & - & - \\
\hline 6.488 & 0.00310 & 0.0620 & $32^{4}$ & 19465 & 1 & 30990 & 65281 & 103060 & - \\
\hline 6.515 & 0.00302 & 0.0604 & $32^{4}$ & 17385 & 1 & 30990 & 140212 & 108530 & - \\
\hline 6.550 & 0.00291 & 0.0582 & $32^{4}$ & 8805 & 1 & 30990 & 136781 & - & - \\
\hline 6.575 & 0.00282 & 0.0564 & $32^{4}$ & 21455 & 1 & 30990 & 144241 & 106750 & - \\
\hline 6.608 & 0.00271 & 0.0542 & $32^{4}$ & 21195 & 1 & 30990 & 171977 & 113920 & - \\
\hline 6.664 & 0.00257 & 0.0514 & $32^{4}$ & 21200 & 1 & 30990 & 94440 & 175500 & - \\
\hline 6.740 & 0.00238 & 0.0476 & $48^{4}$ & 8005 & 1 & - & 88520 & 217740 & 48230 \\
\hline 6.800 & 0.00224 & 0.0448 & $32^{4}$ & 39077 & 1 & 30990 & 110200 & 299550 & 57136 \\
\hline 6.880 & 0.00206 & 0.0412 & $48^{4}$ & 8095 & 1 & - & 110020 & 360690 & 65678 \\
\hline 6.950 & 0.00193 & 0.0386 & $32^{4}$ & 39670 & 1 & 30990 & 117780 & 318700 & 76080 \\
\hline 7.030 & 0.00178 & 0.0356 & $48^{4}$ & 16390 & 1 & - & 96991 & 152330 & 97801 \\
\hline 7.150 & 0.00160 & 0.0320 & $48^{3} \times 64$ & 8094 & 2 & 29620 & 96342 & 163900 & 106150 \\
\hline 7.280 & 0.00142 & 0.0284 & $48^{3} \times 64$ & 7956 & 2 & 37340 & 103748 & 118460 & 110330 \\
\hline 7.373 & 0.00125 & 0.0250 & $48^{3} \times 64$ & 9246 & 2 & 20780 & 116390 & 108100 & 164450 \\
\hline 7.596 & 0.00101 & 0.0202 & $64^{4}$ & 9514 & 2 & 36650 & 120000 & 113510 & 171020 \\
\hline 7.825 & 0.00082 & 0.0164 & $64^{4}$ & 9536 & 2 & 44390 & 119200 & 116070 & 105970 \\
\hline
\end{tabular}

Table III: Parameters used in simulations with the HISQ/tree action on $N_{\tau}=6,810$, and 12 lattices and the LCP defined by $m_{l} / m_{s}=0.05$. The quark masses are given in units of the lattice spacing $a$. The statistics in molecular dynamics time units $T U$ are given for both the zero and finite temperature runs. The column "length" lists the length of the trajectory in TU before the Metropolis accept-reject step for zero-temperature runs. All the finite temperature lattices have trajectories of unit length except for $\beta=5.90$ and 5.95 , where it was $0.5 \mathrm{TU}$. The lattice sizes used for the finite temperature simulations were $24^{3} \times 6,32^{3} \times 8,40^{3} \times 10$, and $48^{3} \times 12$. Measurements were performed after $1 \mathrm{TU}$ on all ensembles, except for the large zero-temperature lattices with length $=2 \mathrm{TU}$, where they were performed every 2 TUs. 


\begin{tabular}{|c|c|c|c|}
\hline$\beta$ & $r_{0} / a$ & $r_{1} / a$ & $r_{0} / r_{1}$ \\
\hline 5.900 & $1.909(11)$ & $1.230(133)$ & $1.552(168)$ \\
6.000 & $2.094(21)$ & $1.386(80)$ & $1.511(89)$ \\
6.050 & $2.194(22)$ & $1.440(31)$ & $1.524(36)$ \\
6.100 & $2.289(21)$ & $1.522(30)$ & $1.504(33)$ \\
6.195 & $2.531(24)$ & $1.670(30)$ & $1.516(31)$ \\
6.285 & $2.750(30)$ & $1.822(30)$ & $1.509(30)$ \\
6.341 & $2.939(11)$ & $1.935(30)$ & $1.519(24)$ \\
6.354 & $2.986(41)$ & $1.959(30)$ & $1.524(31)$ \\
6.423 & $3.189(22)$ & $2.096(21)$ & $1.522(18)$ \\
6.460 & $3.282(32)$ & $2.165(20)$ & $1.516(20)$ \\
6.488 & $3.395(31)$ & $2.235(21)$ & $1.519(20)$ \\
6.550 & $3.585(14)$ & $2.369(21)$ & $1.513(15)$ \\
6.608 & $3.774(20)$ & $2.518(21)$ & $1.499(15)$ \\
6.664 & $3.994(14)$ & $2.644(23)$ & $1.511(14)$ \\
6.740 & $4.293(32)$ & $2.856(11)$ & $1.503(13)$ \\
6.800 & $4.541(30)$ & $3.025(22)$ & $1.501(15)$ \\
6.880 & $4.959(28)$ & $3.265(23)$ & $1.519(14)$ \\
6.950 & $5.249(20)$ & $3.485(22)$ & $1.506(11)$ \\
7.030 & $5.691(32)$ & $3.763(13)$ & $1.512(10)$ \\
7.150 & $6.299(59)$ & $4.212(42)$ & $1.495(20)$ \\
7.280 & $7.140(53)$ & $4.720(33)$ & $1.513(15)$ \\
7.373 & $7.801(79)$ & $5.172(34)$ & $1.508(18)$ \\
7.596 & $9.443(237)$ & $6.336(56)$ & $1.490(40)$ \\
7.825 & $11.51(378)$ & $7.690(58)$ & $1.497(50)$ \\
\hline
\end{tabular}

Table IV: Values of $r_{1}$ and $r_{0}$ in lattice units for different $\beta$

square of the second derivative of the fit function in the considered interval times a real parameter $\mathrm{sm}$. We chose the largest possible value of the smoothing parameter $s m=0.7$ that still gives an acceptable $\chi^{2} /$ dof $=1.13$. To estimate the uncertainties of the spline, we performed a bootstrap analysis. In Fig. 13, we show the $r_{1}$ scale as a function of $\beta$, normalized by the asymptotic twoloop beta function $f(\beta)$. The errors are bootstrap errors. The Allton-type fit and the smoothing spline fits give very similar results as well as uncertainties.

To calculate the EoS, we also need the nonperturbative beta function

$$
R_{\beta}=-a \frac{d \beta}{d a}=\frac{r_{1}}{a}\left(\frac{d\left(r_{1} / a\right)}{d \beta}\right)^{-1} .
$$

Figure 13 shows $R_{\beta}$ obtained from both the Allton-type and smoothing-spline fits, together with bootstrap errors. The fit and the splines agree within the errors. The largest error in $R_{\beta}$ is about $3 \%$. At sufficiently large $\beta$, i.e., close to the continuum limit, $R_{\beta}$ is expected to be given by its asymptotic two-loop form

$$
R_{\beta}^{2-\text { loop }}=20 b_{0}+200 b_{1} / \beta .
$$

The asymptotic limit is approached from below [22], as with the p4 action. However, for the HISQ action, we
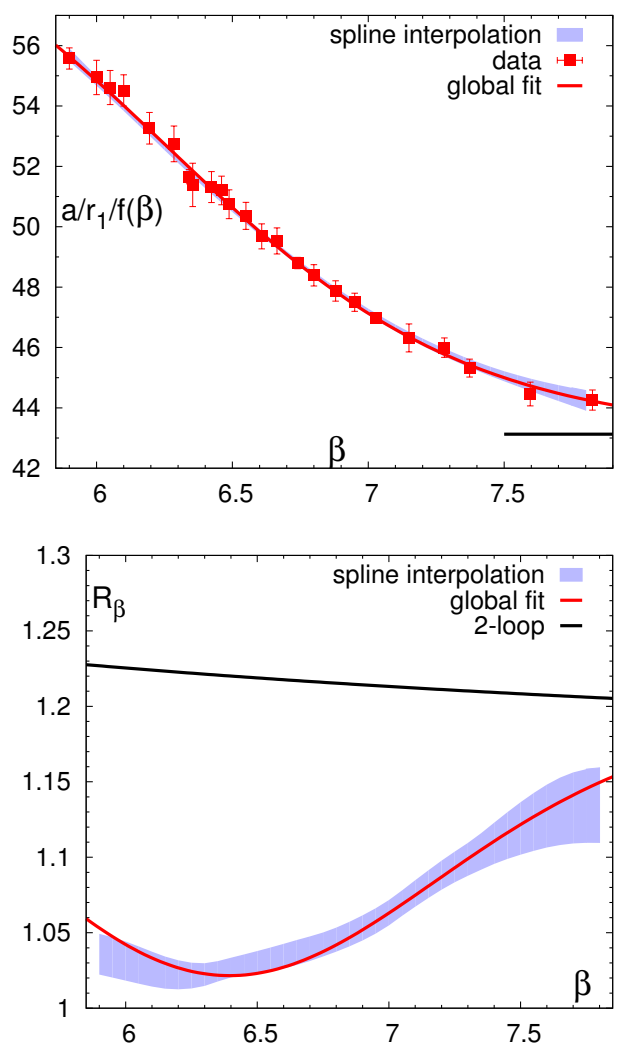

Figure 13: The scale $a / r_{1}$ normalized by the asymptotic twoloop beta function (top) and the nonperturbative beta function, $R_{\beta}$, (bottom) as a function of $\beta$, which has been derived from this using Eq. (B7) and the fit and spline interpolations shown in the left hand figure.

see that the deviations are at most $20 \%$ over the range considered, compared with a factor of two deviation in the case of the p4 action [22]. In our calculations of the EoS we use $R_{\beta}$ obtained from the fit with the Allton-type ansatz.

Finally, we compare the potential calculated at different $\beta$. To do so, we normalize it with an additive constant. We do this by requiring that the potential $V\left(r_{1}\right)=0.2060 / r_{1}$. This normalization condition is equivalent to the one used in Ref. [5]. Here we choose $r_{1}$, because it has smaller errors on fine lattices. The normalized potential in units of $r_{1}$ is plotted in Fig. 14 against the tree-level improvement radius $r \rightarrow r_{I}$, where $r_{I}$ is the improved distance defined from the free lattice gluon propagator [5]. Down to distances $r=0.2 r_{1}$ or $r=0.062 \mathrm{fm}$, we find no significant dependence on the lattice spacing within the estimated errors.

To cross check our determination of the lattice spacing, we also calculated the scale $w_{0}$, defined from the gradient flow [41]. Our results for the $w_{0}$ scale are shown in Fig. 15 in units of $r_{1}$. As above, for $\beta<6.423$ the value of $r_{1}$ was estimated as $r_{0} /\left(r_{0} / r_{1}\right)_{\text {cont }}$. As one can see from the figure, this ratio appears to scale as $a^{2}$ for $\left(a / r_{1}\right)^{2}<0.4$, i.e., for $\beta \geq 6.195$. We per- 


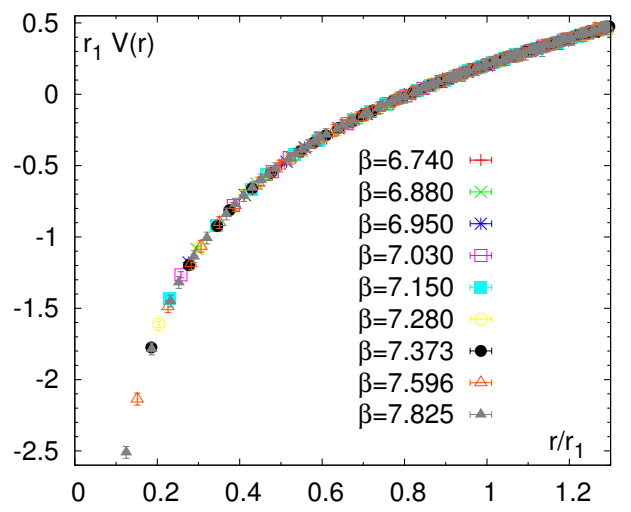

Figure 14: The static potential versus distance in units of $r_{1}$ for different $\beta$. Here we use the improved estimator $r_{I}$ to define the distance $r$.

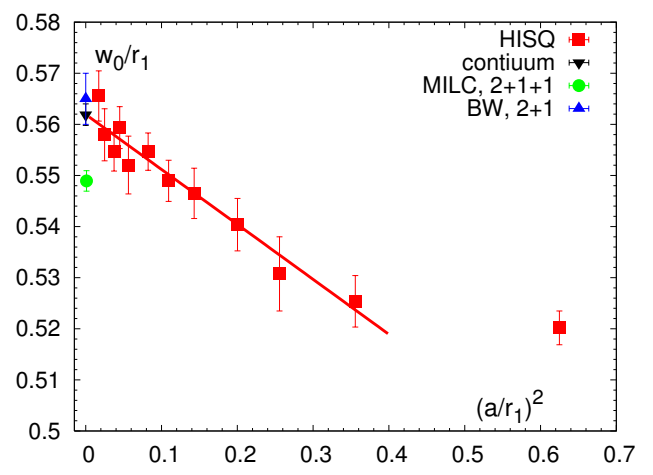

Figure 15: The $w_{0}$ scale as function of the lattice spacing together with the continuum extrapolation. Also shown are results from $2+1$ flavor QCD [41] and 2+1+1 flavor QCD [59].

form a continuum extrapolation of the ratio $w_{0} / r_{1}$ using a simple form $\left(w_{0} / r_{1}\right)_{\text {cont }}+h_{w}\left(a / r_{1}\right)^{2}$. In the continuum limit, we obtain $\left(w_{0} / r_{1}\right)_{\text {cont }}=0.5619(21)$ or $w_{0}=0.1749(14) \mathrm{fm}$. This value agrees with the value quoted in Ref. [41], $w_{0}=0.1755(18)(4) \mathrm{fm}$, within the estimated errors. Our value of $w_{0}$ is higher than the preliminary value reported by MILC $w_{0}=0.1711(2)(8)(2)(3) \mathrm{fm}$ for $2+1+1$ flavor QCD [59]. For the slope parameter we get $h_{w}=-0.1076(149)$ with $\chi^{2} /$ dof $=0.38$. If we use the $w_{0}$ scale instead of the $r_{1}$ scale, the temperature values for $N_{\tau}=8$ lattices for $T<150 \mathrm{MeV}$ would be lower by $6 \%$, and for $N_{\tau}=10$ and 12 calculations the differences in the temperature scale would be only $4 \%$ or less.

\section{Appendix C: Hadronic observables}

\section{Line of constant physics}

It is standard practice to present results for thermodynamic quantities as a function of temperature at fixed, renormalized quark masses. We start by setting a con-

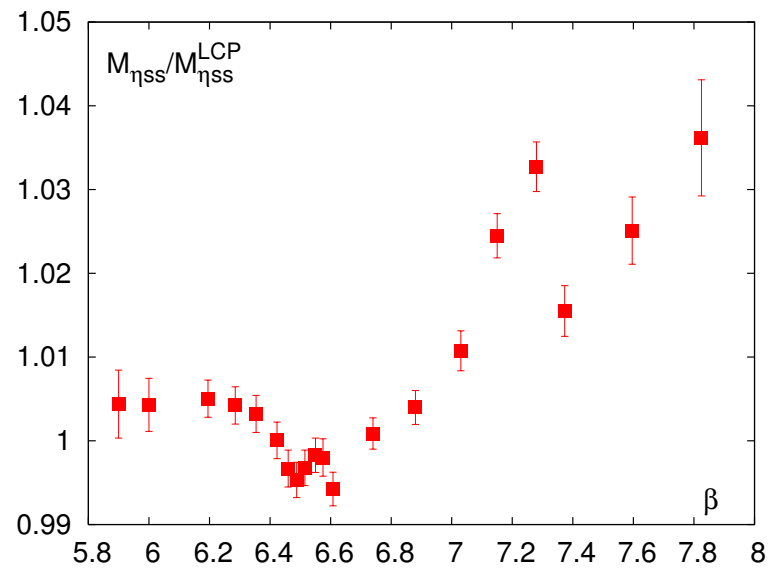

Figure 16: The calculated masses of the $\eta_{s \bar{s}}$ meson normalized by the chosen LCP value $M_{\eta_{s \bar{s}}}=695 \mathrm{MeV}$ as a function of $\beta$.

stant value of the strange quark mass $m_{s}$, preferably, its physical value, and then set the mass of the light quarks to $m_{s} / 20$. We determine the strange quark mass by requiring that the mass of the un-mixed pseudoscalar $s \bar{s}$ meson, $\eta_{s \bar{s}}$ is equal to a prescribed value expressed in units of $r_{1}$. We aim at the value suggested by leading order chiral perturbation theory, where the mass of $\eta_{s \bar{s}}$ meson in terms of the kaon and pion masses is, $M_{\eta_{s \bar{s}}}=$ $\sqrt{2 m_{K}^{2}-m_{\pi}^{2}}=686 \mathrm{MeV}$. In practice, this requires some tuning and, as discussed later, it turns out that our LCP is best described by the value $M_{\eta_{s \bar{s}}}=695 \mathrm{MeV}$. Setting the line of constant physics (LCP) in this way requires a combination of determining the lattice spacing in physical units (see Appendix B) and the hadron spectrum at zero temperature, including, at least, the mass of the $\eta_{s \bar{s}}$, a calculation with costs that mount as the lattice spacing decreases. Thus, some retuning is usually needed to correct for an imprecise determination.

For the present study, we extend the LCP of our previous work [5] to weaker coupling in order to cover the range needed for $N_{\tau}=12$. In our previous work, the hadron spectrum was measured along the LCP up to $\beta=$ 6.8. The masses of the pseudoscalar mesons were also measured at $\beta=7.28$ with the relatively low statistics of about 1,400 equilibrated time units. Here, we added or extended nine $T=0$ ensembles with $6.8<\beta \leq 7.825$ as described in Appendix B. On the extended ensembles, in addition to measuring thermodynamic quantities needed for the zero temperature subtraction, we measured the masses and decay constants of the pseudoscalar mesons and the masses of the vector mesons. These quantities allow us to quantify the lattice artifacts due to taste breaking and can be used as an alternative means to set the lattice spacing, thus providing additional validation of our calculations.

The masses of the three pseudoscalar mesons are given in lattice units in Table V. The mass of the $\eta_{s \bar{s}}$ normal- 


\begin{tabular}{|c|c|c|c|}
\hline$\beta$ & $a M_{\pi}$ & $a M_{K}$ & $a M_{\eta_{s}}$ \\
\hline 5.900 & $0.20162(09)$ & $0.63407(17)$ & $0.86972(11)$ \\
6.000 & $0.18381(37)$ & $0.57532(51)$ & $0.79046(27)$ \\
6.195 & $0.15143(14)$ & $0.47596(16)$ & $0.65506(11)$ \\
6.285 & $0.13823(50)$ & $0.43501(47)$ & $0.59951(28)$ \\
6.354 & $0.12923(15)$ & $0.40628(20)$ & $0.55982(17)$ \\
6.423 & $0.12022(12)$ & $0.37829(19)$ & $0.52161(17)$ \\
6.460 & $0.11528(21)$ & $0.36272(34)$ & $0.50137(32)$ \\
6.488 & $0.11245(15)$ & $0.35313(27)$ & $0.48716(17)$ \\
6.515 & $0.10975(12)$ & $0.34453(29)$ & $0.47516(29)$ \\
6.550 & $0.10629(16)$ & $0.33322(38)$ & $0.45989(24)$ \\
6.575 & $0.10469(68)$ & $0.32521(55)$ & $0.44869(50)$ \\
6.608 & $0.10001(17)$ & $0.31333(28)$ & $0.43286(29)$ \\
6.664 & $0.09572(18)$ & $0.29837(37)$ & $0.41178(32)$ \\
6.740 & $0.087991(64)$ & $0.27735(12)$ & $0.38342(10)$ \\
6.800 & $0.0849(18)$ & $0.26387(99)$ & $0.36257(68)$ \\
6.880 & $0.07714(16)$ & $0.24314(16)$ & $0.33630(11)$ \\
7.030 & $0.06744(15)$ & $0.21202(19)$ & $0.29381(20)$ \\
7.150 & $0.06126(18)$ & $0.19231(20)$ & $0.26631(16)$ \\
7.280 & $0.05516(17)$ & $0.17209(19)$ & $0.23824(18)$ \\
7.373 & $0.04990(22)$ & $0.15530(16)$ & $0.21531(12)$ \\
7.596 & $0.04106(44)$ & $0.12896(30)$ & $0.17810(12)$ \\
7.825 & $0.03425(23)$ & $0.10695(46)$ & $0.14731(15)$ \\
\hline
\end{tabular}

Table V: The pseudoscalar meson masses for the HISQ/tree action along the $m_{l}=0.05 m_{s} \mathrm{LCP}$.

ized by the value $M_{\eta_{s \bar{s}}}=695 \mathrm{MeV}$ used to define the LCP is plotted in Fig. 16 as a function of $\beta$. As one can see, the central values are systematically above the nominal value of $685.8 \mathrm{MeV}$ quoted in Ref. [60]. The average value from ensembles with $\beta<7.03$ is about $695 \mathrm{MeV}$. Therefore, we define our LCP using this value. That is, we choose a strange quark mass that gives $M_{\eta_{s \bar{s}}}=695 \mathrm{MeV}$. The resulting strange quark mass is then about $2.6 \%$ larger than its physical value. For $\beta \leq 7.03$ we find that $M_{\eta_{s \bar{s}}}$ (and, in turn, the strange quark mass) along the LCP agrees with the physical values within $(1-2) \sigma$. For the finest ensembles, $\beta>7.03$, we see a systematic deviation of $M_{\eta_{s \bar{s}}}$ towards higher values - by as much as about $3.5 \%$.

For the calculation of the trace anomaly, we need the strange quark mass $m_{s}$ and its derivative as a function of $\beta$ along the LCP. As we have seen, the strange-quark mass input into the simulation drifts slightly above the LCP. We correct for this drift using lowest order chiral perturbation theory, i.e., we assume that $M_{\eta_{s s}}^{2}$ is proportional to $m_{s}$ and calculate the strange quark mass that gives $M_{\eta_{s \bar{s}}}=695 \mathrm{MeV}$. This corrected value is compared with the value used in the simulations in Fig. 17. For the worst case, $\beta=7.825$, this amounts to lowering $m_{s}$ used by about $7 \%$ from the simulated value. The pion and kaon masses follow a pattern similar to that of the $\eta_{s \bar{s}}$ meson, i.e., they are roughly constant for $\beta<7.03$ and increase for larger beta values by approximately the same fractional amount.

We then fit the product $r_{1} m_{s}^{L C P}$ using a renormalization-group-inspired form

$$
r_{1} m_{s}^{L C P} \equiv \tilde{m}_{s}=r_{1} m^{R G I}\left(\frac{20 b_{0}}{\beta}\right)^{4 / 9} \frac{1+m_{1} \frac{10}{\beta} f^{2}(\beta)+m_{2}\left(\frac{10}{\beta}\right)^{2} f^{2}(\beta)+m_{3} \frac{10}{\beta} f^{4}(\beta)}{1+d m_{1} \frac{10}{\beta} f^{2}(\beta)}
$$

where $f(\beta)$ is the 2-loop beta function given by Eq. B3. For the fit parameters we get

$$
\begin{gathered}
m^{R G I}=0.2609 \pm 0.0030 \\
m_{1}=35600 \pm 6097 \\
m_{2}=-21760 \pm 3202 \\
m_{3}=(2.67 \pm 0.50) \cdot 10^{7} \\
d m_{1}=2420 \pm 1346 \\
\chi^{2} / \text { dof }=0.51 .
\end{gathered}
$$

The resulting fit is shown in Fig. 17 together with a smoothing spline fit to the input strange-quark masses.

\section{Pseudoscalar decay constants}

The decay constants of pseudoscalar mesons can be used to check the lattice scale and to estimate the cutoff effects in the $T=0$ calculations. Results for the decay constants are shown in Table VI.

Since they are quite sensitive to the values of the quark masses, we need to take into account the deviations from the LCP, as well as the fact that even on the LCP our quark masses are slightly heavier than the physical ones. Thus, we need to interpolate/extrapolate in the quark masses. To do this we assume that the pseudoscalar decay constants depend linearly on the sum of the quark masses and use the numerical results given in Table VI to determine the slope for each value of $\beta$. The values of the $\eta_{s \bar{s}}$ meson decay constant $f_{\eta}$ and the kaon decay constant $f_{K}$ have been interpolated to the physical quark 


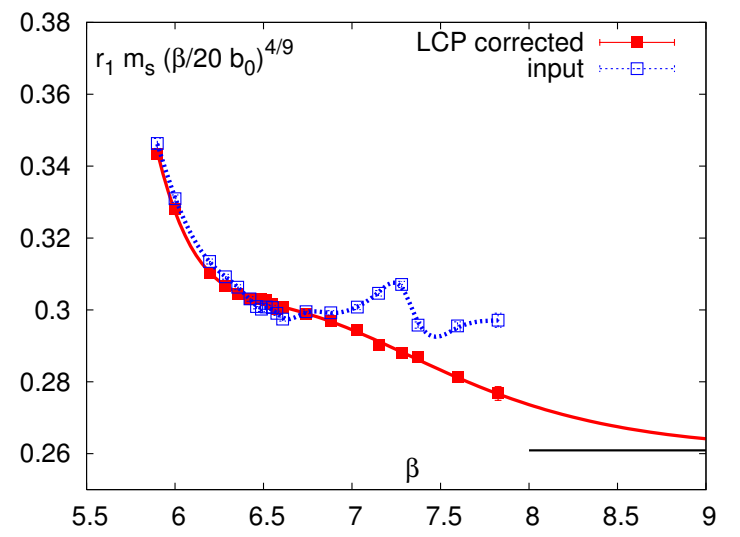

Figure 17: The input strange quark mass and the strange quark mass along LCP together with the respective fits shown as lines. The input quark masses have been fitted with a smooth spline.

\begin{tabular}{|c|c|c|c|c|}
\hline$\beta$ & $a f_{\pi}$ & $a f_{K}$ & $a f_{\eta}$ & $\#$ sources \\
\hline 6.000 & $0.11243(21)$ & $0.13224(31)$ & $0.15290(22)$ & 1 \\
6.195 & $0.09179(21)$ & $0.10835(13)$ & $0.12525(13)$ & 1 \\
6.285 & $0.08366(22)$ & $0.09826(13)$ & $0.11390(13)$ & 1 \\
6.354 & $0.07825(40)$ & $0.09146(19)$ & $0.10598(11)$ & 1 \\
6.423 & $0.07241(18)$ & $0.08515(11)$ & $0.09854(07)$ & 2 \\
6.460 & $0.06885(11)$ & $0.08185(09)$ & $0.09454(08)$ & 4 \\
6.515 & $0.06534(18)$ & $0.07707(15)$ & $0.08946(09)$ & 4 \\
6.575 & $0.06104(49)$ & $0.07265(19)$ & $0.08405(14)$ & 2 \\
6.740 & $0.052190(50)$ & $0.061731(41)$ & $0.071354(27)$ & 2 \\
6.800 & $0.04883(83)$ & $0.05774(18)$ & $0.06717(14)$ & 1 \\
6.880 & $0.045544(67)$ & $0.053749(42)$ & $0.062236(28)$ & 2 \\
7.030 & $0.03951(12)$ & $0.046566(68)$ & $0.054148(43)$ & 2 \\
7.150 & $0.03486(10)$ & $0.041636(68)$ & $0.048654(42)$ & 2 \\
7.280 & $0.03067(13)$ & $0.036894(64)$ & $0.043237(38)$ & 2 \\
7.373 & $0.02787(20)$ & $0.033825(82)$ & $0.039609(43)$ & 2 \\
7.596 & $0.02224(20)$ & $0.02741(21)$ & $0.032344(59)$ & 2 \\
7.825 & $0.01756(32)$ & $0.022526(85)$ & $0.026808(51)$ & 2 \\
\hline
\end{tabular}

Table VI: Results for decay constants of the pseudoscalar mesons in lattice units for the HISQ/tree action along the $m_{l}=0.05 m_{s}$ LCP. We use the normalization in which $f_{\pi} \sim 90$ $\mathrm{MeV}$. In the last column, we list the number of source points used on each configuration to increase the statistics.

masses using this slope. The results are shown in Fig. 18 in units of $r_{1}$. The kaon decay constant has large finite size errors at the two smallest lattices spacings. Therefore, we do not include the corresponding data in the fit. We extrapolate the values of $r_{1} f_{\eta}$ and $r_{1} f_{K}$ to zero lattice spacing assuming a simple form

$$
f_{i} r_{1}=\left(f_{i} r_{1}\right)^{\text {cont }}+e_{i}\left(a / r_{1}\right)^{2}, \quad i=K, \eta .
$$

For the kaon decay constant we get $\left(r_{1} f_{K}\right)^{\text {cont }}=$ $0.17186(24)$ and $e_{K}=0.0230(11)$ with $\chi^{2} /$ dof $=$ 1.20. For the $\eta_{s \bar{s}}$ decay constant we get $\left(r_{1} f_{\eta}\right)^{\text {cont }}=$

\begin{tabular}{|c|c|c|c|}
\hline$\beta$ & $a M_{\rho}$ & $a M_{K^{*}}$ & $a M_{\phi}$ \\
\hline 6.195 & $0.7562(36)$ & $0.8842(18)$ & $1.0050(93)$ \\
6.354 & $0.6375(35)$ & $0.7499(26)$ & $0.8523(08)$ \\
6.423 & $0.6047(43)$ & $0.6950(22)$ & $0.7925(08)$ \\
6.460 & $0.5784251)$ & $0.6709(43)$ & $0.7644(22)$ \\
6.488 & $0.5647(24)$ & $0.6478(22)$ & $0.7363(07)$ \\
6.550 & $0.5324(24)$ & $0.6118(20)$ & $0.6929(14)$ \\
6.608 & $0.5072(39)$ & $0.5757(08)$ & $0.6523(10)$ \\
6.664 & $0.4732(43)$ & $0.5501(26)$ & $0.6180(10)$ \\
6.740 & $0.4286(31)$ & $0.4996(22)$ & $0.5732(05)$ \\
6.880 & $0.2828(489)$ & $0.4359(17)$ & $0.5000(04)$ \\
7.030 & $0.2937(326)$ & $0.3750(126)$ & $0.4333(09)$ \\
7.150 & $0.2866(108)$ & $0.3387(107)$ & $0.3901(15)$ \\
7.280 & $0.2535(96)$ & $0.3026(20)$ & $0.3467(25)$ \\
7.373 & $0.2363(119)$ & $0.2774(33)$ & $0.3165(06)$ \\
7.596 & $0.1923(61)$ & $0.2272(25)$ & $0.2593(15)$ \\
7.825 & $0.1543(120)$ & $0.1884(57)$ & $0.2140(19)$ \\
\hline
\end{tabular}

Table VII: Masses of the vector mesons in lattice units.

$0.19930(24)$ and $e_{\eta}=0.024(12)$ with $\chi^{2} /$ dof $=0.77$. The continuum extrapolation is also shown in Fig. 18, where we compare it with the value of $r_{1} f_{\eta}$ quoted in Ref. [60], and find reasonable agreement. The "PDG" value plotted there is based on the PDG value of $f_{\pi}$ and the value of $f_{K} / f_{\pi}=1.194(5)$ from the recent FLAG review [61], which gives $f_{K}=155.7(9) / \sqrt{2} \mathrm{MeV}$. We find agreement within estimated errors.

\section{Vector meson masses}

The masses of the three vector mesons $\rho, K^{*}$, and $\phi$ are listed in Table VII. All three masses are adjusted to the LCP in a manner similar to the decay constants. For $\rho$ and $K^{*}$ the contribution of an excited state of the same parity is significant in the available temporal range; however, fits with an excited state typically yield low confidence levels. Therefore, in reporting masses on our finest lattices we take, as a systematic error, the difference in the fitted masses with and without an excited state of the same parity. This error is combined linearly with the statistical error in the table.

In Fig. 19, we show the $\phi$ meson mass $m_{\phi}$ in $r_{1}$ units as a function of the lattice spacing together with the continuum extrapolation. Again, we use the simple form $\left(r_{1} m_{\phi}\right)_{\text {cont }}+g_{\phi}\left(a / r_{1}\right)^{2}$ to do the continuum extrapolations, and get $\left(r_{1} m_{\phi}\right)=1.5961(30)$ and $g_{\phi}=0.236(18)$ with $\chi^{2} /$ dof $=0.42$. Our continuum extrapolation agrees with the experimental result (shown as the band).

In summary, as one can see from Figs. 18 and 19, the hadronic observables provide additional valuable crosschecks for the determination of the lattice spacing. The cutoff dependence of $f_{K}$ and $m_{\phi}$ is very similar to the cutoff dependence of $w_{0}$. Therefore, the change in the 

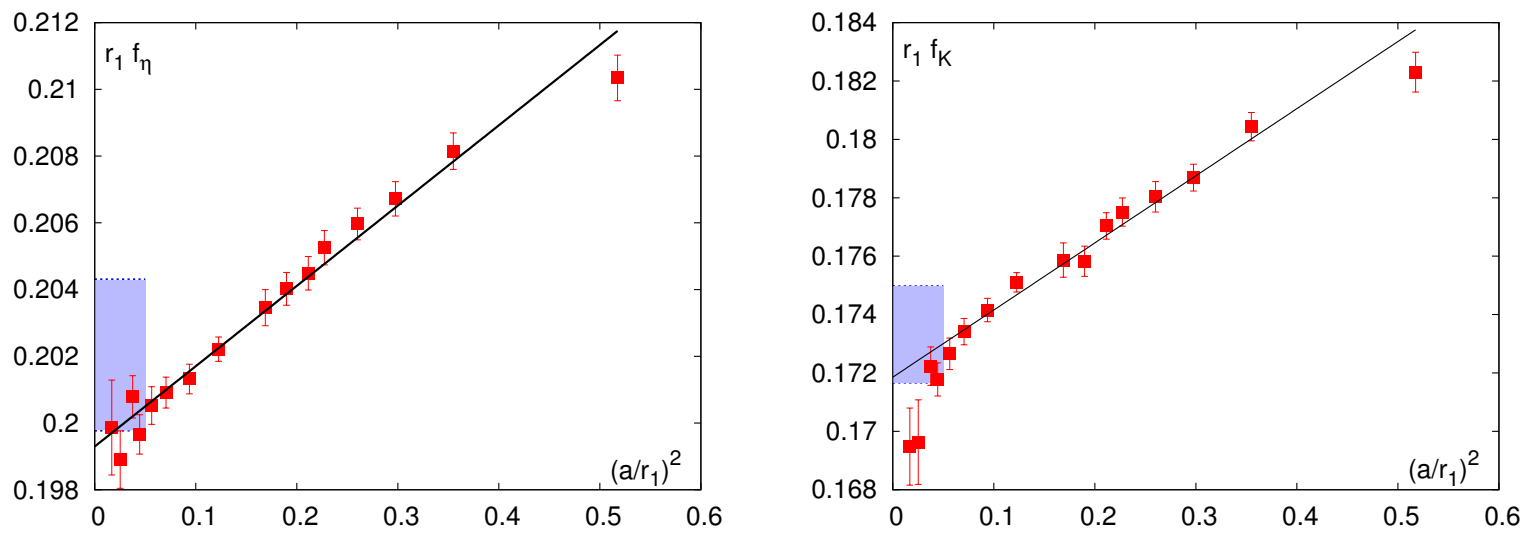

Figure 18: The decay constants of $\eta_{s \bar{s}}$ meson (left) and kaon (right) together with the continuum extrapolations. The data are corrected for deviations from the LCP as described in the text. The bands show the value of these decay constants from Ref. [60] and PDG. Here we use the value $r_{1}=0.3106(14)(8)(4) \mathrm{fm}$. The errors on $r_{1}$ have been added linearly and combined with the errors on $f_{\eta}$ and $f_{K}$ in quadratures.

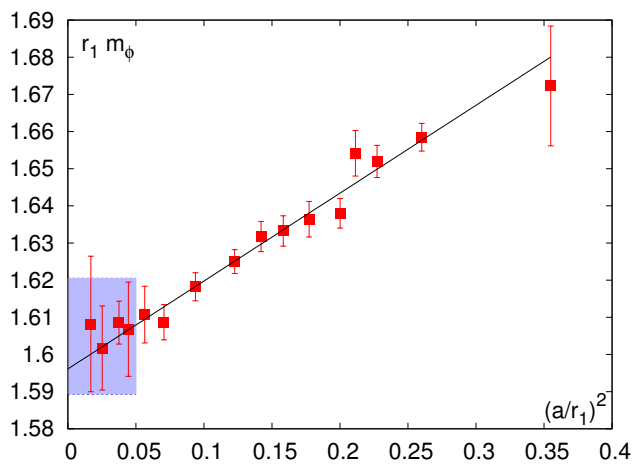

Figure 19: The $\phi$ meson mass as function of the lattice spacing together with the continuum extrapolation. The data are corrected for deviations from the LCP as described in the text. The band shows the experimental value.

lattice spacing and the temperature scale will be similar to the case when $w_{0}$ is used to set the lattice spacing.

\section{Appendix D: Observables for EoS}

In this appendix, we summarize the quantities we used to evaluate the trace anomaly. They include the expectation values of local observables such as the gauge action density $s_{G}$ and the light and strange quark condensates. At nonzero temperature, we also report results for the disconnected light and strange chiral susceptibilities $\left(\chi_{l}^{\text {disc }}\right.$ and $\left.\chi_{s}^{\text {disc }}\right)$, i.e., the fluctuations of the light and strange quark condensates, as well as the bare Polyakov loop $L_{\text {bare }}$. We use the same definitions of these quantities as in Ref. [5]. In particular, the quark condensates are normalized per single flavor, the disconnected chiral susceptibility for light quarks is normalized for two flavors, and the disconnected chiral susceptibility for strange quarks is normalized for a single flavor.

The gauge action density and the quark condensates for zero temperature are given in Table VIII. The observables at nonzero temperature are summarized in Tables IX, X, XI, and XII for $N_{\tau}=6,8,10$, and 12 lattices.

For the calculation of the trace anomaly, one also needs the lattice spacing in units of $r_{1}$, the nonperturbative beta function $R_{\beta}$, the strange quark mass as function of $\beta$ along the LCP and the mass renormalization function $R_{m}$ calculated along the LCP. The parametrization of $r_{1} / a$ and $R_{\beta}$ and the calculation of their errors have been discussed in Appendix B. In Table VIII, we give these quantities with their errors. The value of $m_{s}$ along the LCP has been discussed in Appendix C, where an explicit parametrization of $\tilde{m}_{s}=r_{1} m_{s}^{L C P}$ has been given. The mass renormalization function $R_{m}$ can be written as

$$
R_{m}=-R_{\beta}^{-1}\left(1-\tilde{R}_{m} R_{\beta}\right), \quad \tilde{R}_{m}=\frac{1}{\tilde{m}_{s}} \frac{d \tilde{m}_{s}}{d \beta} .
$$

The values of $\tilde{m}_{s}$ and $\tilde{R}_{m} R_{\beta}$ are also given in Table VIII. As one can see from Figs. 16 and 17, the deviations of the input strange quark masses from the LCP for $\beta \geq 7.03$ are at most $7 \%$ and are below $1 \%$ for $\beta<7.03$. To include the errors arising from the deviations from the LCP, we assign a $1 \%$ error to $m_{s}$ for $\beta<7.03$ and $10 \%$ errors to $m_{s}$ for $\beta \geq 7.03$ in Eq. (9). All the errors discussed above are added in quadratures to get the the total error estimate of the trace anomaly presented in Sec. III. Our estimate of the systematic errors on the trace anomaly due to the deviations from LCP includes only the difference between the input $m_{s}$ and the value of $m_{s}$ along the LCP. Since $m_{l} / m_{s}$ is kept constant, there is no additional uncertainty due to $m_{l}$. The value of $m_{s}$, however, will affect the expectation value of the gluon action and the quark condensates shown in Eqs. $(8,9)$. We did not estimate these effects, but based on the past experience $[21,24]$ we expect that these will be small. 


\begin{tabular}{|c|c|c|c|c|c|c|c|}
\hline$\beta$ & $\left\langle s_{G}\right\rangle$ & $\langle\bar{\psi} \psi\rangle_{l}$ & $\langle\bar{\psi} \psi\rangle_{s}$ & $r_{1} / a$ & $R_{\beta}$ & $\tilde{m}_{s}$ & $R_{\beta} R_{m}$ \\
\hline 5.900 & $2.632783(101)$ & $0.049104(40)$ & $0.105812(26)$ & $1.264(5)$ & $1.048(17)$ & $0.16563(166)$ & $-1.6999(114)$ \\
\hline 5.950 & $2.597143(81)$ & $0.043881(54)$ & $0.098036(32)$ & $1.325(5)$ & $1.043(17)$ & $0.16060(161)$ & $-1.5925(97)$ \\
\hline 6.000 & $2.561602(72)$ & $0.038908(37)$ & $0.090108(26)$ & $1.390(4)$ & $1.038(16)$ & $0.15646(156)$ & $-1.4972(77)$ \\
\hline 6.025 & $2.544156(82)$ & $0.036579(45)$ & $0.086515(29)$ & $1.424(4)$ & $1.036(15)$ & 0.15468 & $-1.4545(66)$ \\
\hline 6.050 & $2.526767(19)$ & $0.034355(45)$ & $0.083052(30)$ & $1.459(4)$ & $1.034(15)$ & 0.15306 & $-1.4149(60)$ \\
\hline 6.075 & $2.509536(128)$ & $0.032248(36)$ & $0.079962(31)$ & 194( 4) & $1.032(14)$ & 0.15160 & $3(51)$ \\
\hline 6.100 & $2.492425(78)$ & $0.030236(36)$ & $0.076478(23)$ & (4) & 1.030 & 0.1502 & $(47)$ \\
\hline 6.125 & 2.475464 & 0.028340 & 733 & $(4)$ & 1.02 & 9) & 40) \\
\hline 6.150 & 2.458696 & 0.026433 & 0.070257 & $1.607(4)$ & 3) & 0.1 & -1.28 \\
\hline 6.175 & $2.442094(39)$ & 0.0 & 3) & $646(4)$ & 2) & 17) & -1.2 \\
\hline 6.195 & $2.429035(30)$ & 0.023470 & 2) & $9(4)$ & 2) & 0.1 & -1.2 \\
\hline 6.245 & $2.396804(11)$ & $0.020449(14)$ & $9664(10)$ & $2(4)$ & 1.02 & 0.1447 & -1.20 \\
\hline 6.285 & 2.371682 & 0.0183176 & $0.0557043(69)$ & (4) & $1.022(9)$ & 0.1 & -1.1 \\
\hline 6.341 & 2.33743 & 0.0156426 & 0.0506613 & $1.936(4)$ & $1.021(8)$ & 0.14 & 12) \\
\hline 6.354 & $2.3297339(226)$ & $0.0151142(157)$ & $0.0495513(82)$ & $1.961(4)$ & $1.021(8)$ & 0.1 & 12) \\
\hline 6.390 & 2.3084526 & $0.0135987(151)$ & $0.0464432(90)$ & $2.031(4)$ & $1.021(8)$ & 0.1 & -1.1 \\
\hline 6.423 & $2.2894362(269)$ & $0.0124345(110)$ & $0.0440691(80)$ & $2.098(5)$ & $1.022(8)$ & 0.14 & $(10)$ \\
\hline 6.460 & $2.2685484(184)$ & $0.0111926(157)$ & $0.0413543(72)$ & $2.175(5)$ & $1.022(8)$ & 0.14008 & $-1.1223(10)$ \\
\hline 6.488 & $2.2530949(69)$ & $0.0103368(80)$ & $0.0395263(45)$ & $2.235(5)$ & $1.023(9)$ & 0.139 & $-1.1186(10)$ \\
\hline 6.515 & $2.2384913(119)$ & $0.0096409(162)$ & $0.0379912(95)$ & $2.295(5)$ & $1.024(9)$ & 0.1391 & -1.1 \\
\hline 6.550 & $2.2198533(261)$ & $0.0087186(96)$ & $0.0359905(69)$ & $2.375(5)$ & $1.026(10)$ & 0.13864 & $-1.1141(11)$ \\
\hline 6.575 & $2.2068224(107)$ & $0.0081675(80)$ & $0.0345257(68)$ & $2.434(5)$ & $1.027(10)$ & 0.13826 & $-1.1134(11)$ \\
\hline 6.608 & $2.1899477(85)$ & $0.0074639(60)$ & $0.0327412(57)$ & $2.513(5)$ & $1.029(10)$ & $0.13776(138)$ & $-1.1134(11)$ \\
\hline 6.664 & $2.1620782(110)$ & $0.0064352(101)$ & $0.0302931(50)$ & $2.654(5)$ & $1.033(10)$ & $0.13691(137)$ & $-1.1150(11)$ \\
\hline 6.740 & $2.1257817(133)$ & $0.0053945(96)$ & $0.0272312(48)$ & $2.856(5)$ & $1.039(10)$ & $0.13574(136)$ & $-1.1196(12)$ \\
\hline 6.800 & $2.0982834(130)$ & $0.0045273(53)$ & $0.0250900(34)$ & $3.026(6)$ & $1.045(10)$ & $0.13479(135)$ & $-1.1244(12)$ \\
\hline 6.880 & $2.0630924(76)$ & $0.0038178(57)$ & $0.0224907(35)$ & $3.266(7)$ & $1.053(10)$ & $0.13348(133)$ & $-1.1313(12)$ \\
\hline 6.950 & $2.0336080(100)$ & $0.0030671(68)$ & $0.0206297(36)$ & $3.491(7)$ & $1.061(10)$ & $0.13230(132)$ & $-1.1373(13)$ \\
\hline 7.030 & $2.0012582(67)$ & $0.0027057(50)$ & $0.0186364(23)$ & $3.764(8)$ & $1.071(10)$ & $0.13091(1309)$ & $-1.1435(13)$ \\
\hline 7.150 & $1.9552310(112)$ & $0.0021015(39)$ & $0.0162827(22)$ & $4.209(11)$ & $1.085(11)$ & $0.12878(1288)$ & $-1.1507(15)$ \\
\hline 7.280 & $1.9083452(117)$ & $0.0015991(68)$ & $0.0140717(28)$ & $4.743(13)$ & $1.101(11)$ & $0.12646(1265)$ & $-1.1552(16)$ \\
\hline 7.373 & $1.8765238(46)$ & $0.0012932(102)$ & $0.0122227(24)$ & $5.160(15)$ & $1.112(12)$ & $0.12481(1248)$ & $-1.1562(17)$ \\
\hline 7.596 & $1.8053831(35)$ & $0.0008701(71)$ & $0.0095488(14)$ & $6.297(24)$ & $1.135(21)$ & $0.12103(1210)$ & $-1.1524(28)$ \\
\hline 7.825 & $1.7388070(42)$ & $0.0005625(54)$ & $0.0075428(23)$ & $7.696(51)$ & $1.154(27)$ & $0.11751(1175)$ & $-1.1420(33)$ \\
\hline
\end{tabular}

Table VIII: The gauge action density, light and strange quark condensates at zero temperatures. Also shown are the values of $r_{1} / a, \tilde{m}_{s}, R_{\beta}$ and $R_{\beta} R_{m}$ used in the calculation of the trace anomaly along the LCP. 


\begin{tabular}{|c|c|c|c|c|c|c|c|}
\hline$\beta$ & $T[\mathrm{MeV}]$ & $\left\langle s_{G}\right\rangle$ & $\langle\bar{\psi} \psi\rangle_{l}$ & $\langle\bar{\psi} \psi\rangle_{s}$ & $\chi_{l}^{d i s c}$ & $\chi_{s}^{d i s c}$ & $L_{\text {bare }}$ \\
\hline 5.900 & 133.8 & $2.632253(64)$ & $0.044948(25)$ & $0.105106(15)$ & $0.577(10)$ & $0.0644(17)$ & $0.002599(20)$ \\
\hline 5.950 & 140.3 & $2.596445(58)$ & $0.038962(28)$ & $0.097108(17)$ & $0.655(12)$ & $0.0681(19)$ & $0.003157(22)$ \\
\hline 6.000 & 147.2 & $2.560827(68)$ & $0.032932(30)$ & $0.088920(16)$ & $0.741(11)$ & $0.0704(14)$ & $0.004077(20)$ \\
\hline 6.025 & 150.8 & $2.543240(18)$ & $0.029995(27)$ & $0.085152(10)$ & $0.810(16)$ & $0.0711(15)$ & $0.004600(32)$ \\
\hline 6.050 & 154.5 & $2.525666(19)$ & $0.027029(32)$ & $0.081458(18)$ & $0.946(26)$ & $0.0754(27)$ & $0.005294(33)$ \\
\hline 6.075 & 158.2 & $2.508305(33)$ & $0.024144(50)$ & $0.078122(26)$ & $1.061(19)$ & $0.0777(21)$ & $0.006013(28)$ \\
\hline 6.100 & 162.1 & $2.490962(34)$ & $0.020983(31)$ & $0.074290(16)$ & $1.148(23)$ & $0.0798(18)$ & $0.007027(18)$ \\
\hline 6.125 & 166.0 & $2.473804(31)$ & $0.017975(47)$ & $0.070757(17)$ & $1.223(24)$ & $0.0816(29)$ & $0.008156(31)$ \\
\hline 6.150 & 170.1 & $2.456840(33)$ & $0.014837(53)$ & $0.067264(24)$ & $1.313(28)$ & $0.0889(19)$ & $0.009463(53)$ \\
\hline 6.175 & 174.3 & $2.439980(29)$ & $0.012008(39)$ & $0.063819(18)$ & $1.148(26)$ & $0.0906(31)$ & $0.010942(55)$ \\
\hline 6.195 & 177.8 & $2.426644(30)$ & $0.009958(46)$ & $0.061004(18)$ & $1.009(22)$ & $0.0932(15)$ & $0.012192(42)$ \\
\hline 6.245 & 186.5 & $2.394214(29)$ & $0.006302(37)$ & $0.054910(31)$ & $0.440(12)$ & $0.0866(23)$ & $0.015619(71)$ \\
\hline 6.285 & 194.1 & $2.368816(49)$ & $0.004572(23)$ & $0.050296(23)$ & $0.1963(71)$ & $0.0753(28)$ & $0.018535(49)$ \\
\hline 6.341 & 205.0 & $2.334602(54)$ & $0.003229(14)$ & $0.044690(24)$ & $0.0686(34)$ & $0.0724(20)$ & $0.022822(75)$ \\
\hline 6.354 & 207.6 & $2.326948(71)$ & $0.0030128(68)$ & $0.043460(18)$ & $0.05033(157)$ & $0.06293(215)$ & $0.023805(68)$ \\
\hline 6.423 & 222.1 & $2.286667(62)$ & $0.0022268(37)$ & $0.037597(16)$ & $0.01467(44)$ & $0.04245(122)$ & $0.029564(72)$ \\
\hline 6.488 & 236.6 & $2.250583(43)$ & $0.0018235(37)$ & $0.033135(14)$ & $0.00656(46)$ & $0.02796(45)$ & $0.034892(104)$ \\
\hline 6.515 & 243.0 & $2.236043(44)$ & $0.0017067(23)$ & $0.031671(12)$ & $0.00394(25)$ & $0.02268(48)$ & $0.037358(81)$ \\
\hline 6.550 & 251.4 & $2.217540(36)$ & $0.0015783(23)$ & $0.029853(10)$ & $0.00289(28)$ & $0.01663(45)$ & $0.040428(102)$ \\
\hline 6.575 & 257.7 & $2.204590(57)$ & $0.0014903(19)$ & $0.028517(10)$ & $0.00186(20)$ & $0.01368(46)$ & $0.042698(84)$ \\
\hline 6.608 & 266.1 & $2.187842(66)$ & $0.0013966(12)$ & $0.026962(8)$ & $0.00166(13)$ & $0.01048(32)$ & $0.045373(81)$ \\
\hline 6.664 & 281.0 & $2.160154(56)$ & $0.00127253(92)$ & $0.0249128(67)$ & $0.000646(55)$ & $0.006665(202)$ & $0.050566(88)$ \\
\hline 6.800 & 320.4 & $2.096708(55)$ & $0.00104636(41)$ & $0.0207485(33)$ & $0.000170(48)$ & $0.002140(77)$ & $0.062640(66)$ \\
\hline 6.950 & 369.6 & $2.032442(34)$ & $0.00086791(28)$ & $0.0172826(20)$ & $0.000055(14)$ & $0.000795(36)$ & $0.076417(96)$ \\
\hline 7.150 & 445.6 & $1.954433(30)$ & $0.000696540(50)$ & $0.01390563(83)$ & $0.00000214(44)$ & $0.0002043(52)$ & $0.094300(69)$ \\
\hline 7.280 & 502.1 & $1.907706(57)$ & $0.000609280(48)$ & $0.01216886(50)$ & $0.00000388(166)$ & $0.0001201(108)$ & $0.105861(91)$ \\
\hline 7.373 & 546.3 & $1.875942(38)$ & $0.000531641(23)$ & $0.01062292(55)$ & $0.000001297(43)$ & $0.0002442(32)$ & $0.113932(98)$ \\
\hline 7.596 & 666.7 & $1.805019(28)$ & $0.000422615(31)$ & $0.00844724(40)$ & $0.000001080(397)$ & $0.0001474(113)$ & $0.132910(111)$ \\
\hline 7.825 & 814.8 & $1.738571(16)$ & $0.000338793(10)$ & $0.00677357(19)$ & $0.0000003877(22)$ & $0.00007679(64)$ & $0.151621(112)$ \\
\hline
\end{tabular}

Table IX: Expectation value of local observables calculated on $N_{\tau}=6$ lattices 


\begin{tabular}{|c|c|c|c|c|c|c|c|}
\hline$\beta$ & $T[\mathrm{MeV}]$ & $\left\langle s_{G}\right\rangle$ & $\langle\bar{\psi} \psi\rangle_{l}$ & $\langle\bar{\psi} \psi\rangle_{s}$ & $\chi_{l}^{\text {disc }}$ & $\chi_{s}^{d i s c}$ & $L_{\text {bare }}$ \\
\hline 6.050 & 115.8 & $2.5266237(348)$ & $0.0327762(186)$ & $0.0828810(115)$ & $0.4139(69)$ & $0.05669(150)$ & $0.0004353(134)$ \\
\hline 6.125 & 124.5 & $2.4753158(262)$ & $0.0264181(110)$ & $0.0730705(82)$ & $0.4706(75)$ & $0.06081(109)$ & $0.0006204(47)$ \\
\hline 6.175 & 130.7 & $2.4419268(287)$ & $0.0225733(227)$ & $0.0669802(122)$ & $0.4779(81)$ & 05382(134) & $0.0008371(116)$ \\
\hline 6.195 & 133.3 & $2.4288377(376)$ & $0.0211403(154)$ & $0.0645320(91)$ & $0.4973(132)$ & $0.05412(245)$ & $0.0009414(166)$ \\
\hline 6.245 & 139.9 & $2.3965418(294)$ & $0.0177511(150)$ & $0.0591829(61)$ & $0.5346(109)$ & $0.05172(93)$ & $0.0012420(117)$ \\
\hline 6.285 & 145.5 & $2.3713746(283)$ & $0.0151984(242)$ & $0.0550896(84)$ & $0.5607(334)$ & $0.04938(161)$ & $0.0016083(177)$ \\
\hline 6.341 & 153.7 & $2.3370256(418)$ & $0.0118052(185)$ & $0.0498389(77)$ & $0.7507(139)$ & $0.05228(141)$ & $0.0022252(131)$ \\
\hline 6.354 & 155.7 & 2.3292352(137) & $0.0109211(513)$ & $0.0485851(197)$ & $0.8123(357)$ & $0.05624(304)$ & $0.0024708(325)$ \\
\hline 6.390 & 161.3 & 2.3079034(111) & $0.0088051(453)$ & $0.0452635(142)$ & $0.8915(238)$ & $0.05593(184)$ & $0.0031329(387)$ \\
\hline 6.423 & 166.6 & $2.2887805(179)$ & $0.0069624(573)$ & $0.0426307(216)$ & $0.9083(238)$ & $0.05822(342)$ & $0.0038408(355)$ \\
\hline 6.460 & 172.7 & $2.2678076(214)$ & $0.0050971(363)$ & $0.0395899(152)$ & $0.6520(254)$ & $0.05707(351)$ & $0.0048401(370)$ \\
\hline 6.488 & 177.5 & $2.2523061(146)$ & $0.0040973(324)$ & $0.0375676(211)$ & $0.4791(204)$ & $0.05586(308)$ & $0.0055937(523)$ \\
\hline 6.515 & 182.2 & $2.2376344(146)$ & $0.0033605(111)$ & $0.0358104(81)$ & $0.3208(65)$ & $0.05421(204)$ & $0.0064548(245)$ \\
\hline 6.550 & 188.6 & $2.2189982(111)$ & $0.0026714(70)$ & $0.0335964(50)$ & $0.1676(32)$ & $0.04600(150)$ & $0.0076062(174)$ \\
\hline 6.575 & 193.3 & $2.2059639(144)$ & $0.0023153(61)$ & $0.0319944(50)$ & $0.1061(20)$ & $0.04065(91)$ & $0.0084632(181)$ \\
\hline 6.608 & 199.5 & $2.1890447(95)$ & $0.0019665(55)$ & $0.0300405(69)$ & $0.0554(18)$ & $0.03770(106)$ & $0.0096959(240)$ \\
\hline 6.664 & 210.7 & $2.1612301(160)$ & $0.00161467(242)$ & $0.0274505(65)$ & $0.02910(72)$ & $0.028704(850)$ & $0.0118668(196)$ \\
\hline 6.740 & 226.8 & $2.1249421(157)$ & $0.00132194(152)$ & $0.0243573(47)$ & $0.01123(37)$ & $0.017709(487)$ & $0.0150425(309)$ \\
\hline 6.800 & 240.3 & $2.0975154(118)$ & $0.00117166(80)$ & $0.0223027(32)$ & $0.00606(20)$ & $0.010758(192)$ & $0.0176361(287)$ \\
\hline 6.880 & 259.3 & $2.0624169(93)$ & $0.00102161(56)$ & $0.0199079(30)$ & $0.00282(16)$ & $0.005774(105)$ & $0.0213349(344)$ \\
\hline 6.950 & 277.2 & $2.0329709(115)$ & $0.00092680(48)$ & $0.0182584(25)$ & $0.00138(13)$ & $0.003370(64)$ & $0.0246964(418)$ \\
\hline 7.030 & 298.9 & $2.0006938(123)$ & $0.00083316(37)$ & $0.0165135(12)$ & $0.00080(21)$ & $0.001763(20)$ & $0.0286964(278)$ \\
\hline 7.150 & 334.2 & $1.9547446(127)$ & $0.00072858(127)$ & $0.014507068(795)$ & $0.0001447(228)$ & $0.0007811(177)$ & $0.0349571(493)$ \\
\hline 7.280 & 376.6 & $1.9079588(118)$ & $0.00063333(87)$ & $0.012635689(523)$ & $0.0000317(121)$ & $0.0003433(117)$ & $0.0419502(385)$ \\
\hline 7.373 & 409.7 & $1.8762085(84)$ & $0.00055109(22)$ & $0.011005082(305)$ & $0.0000075(24)$ & $0.0004985(47)$ & $0.0470148(330)$ \\
\hline 7.596 & 500.0 & $1.8051472(106)$ & $0.00043608(17)$ & $0.008714430(192)$ & $0.0000040(28)$ & $0.0002289(36)$ & $0.0595132(289)$ \\
\hline 7.825 & 611.1 & $1.73864086(834)$ & $0.0003485897(29)$ & $0.006968632(67)$ & $0.000000600(3)$ & $0.00011855(51)$ & $0.0725663(201)$ \\
\hline
\end{tabular}

Table X: Expectation value of local observables calculated on $N_{\tau}=8$ lattices

\begin{tabular}{|c|c|c|l|l|l|l|l|}
\hline$\beta$ & $T[\mathrm{MeV}]$ & \multicolumn{1}{c|}{$\left\langle s_{G}\right\rangle$} & \multicolumn{1}{c|}{$\langle\bar{\psi} \psi\rangle_{l}$} & \multicolumn{1}{c|}{$\langle\bar{\psi} \psi\rangle_{s}$} & \multicolumn{1}{c|}{$\chi_{l}^{\text {disc }}$} & \multicolumn{1}{c|}{$\chi_{s}^{\text {disc }}$} & \multicolumn{1}{c|}{$L_{\text {bare }}$} \\
\hline 6.488 & 142.0 & $2.2529939(94)$ & $0.008717(66)$ & $0.0392107(30)$ & $0.4696(80)$ & $0.03671(59)$ & $0.0006332(54)$ \\
6.515 & 145.8 & $2.2383351(92)$ & $0.007845(53)$ & $0.0376092(21)$ & $0.4983(48)$ & $0.03601(67)$ & $0.0007530(46)$ \\
6.575 & 154.6 & $2.2066486(87)$ & $0.005916(112)$ & $0.0339874(45)$ & $0.6199(111)$ & $0.03566(87)$ & $0.0011236(72)$ \\
6.608 & 159.6 & $2.1897092(97)$ & $0.004863(141)$ & $0.0320595(57)$ & $0.6914(140)$ & $0.03790(54)$ & $0.0014074(111)$ \\
6.664 & 168.6 & $2.1618075(67)$ & $0.003366(119)$ & $0.0293881(48)$ & $0.5971(104)$ & $0.03805(76)$ & $0.0020061(99)$ \\
6.740 & 181.4 & $2.1254552(49)$ & $0.002058(44)$ & $0.0260341(30)$ & $0.2350(27)$ & $0.03178(63)$ & $0.0030709(50)$ \\
6.800 & 192.2 & $2.0979463(46)$ & $0.001551(21)$ & $0.0237258(22)$ & $0.10175(85)$ & $0.02664(27)$ & $0.0040471(52)$ \\
6.880 & 207.5 & $2.0627661(47)$ & $0.001196(11)$ & $0.0210031(13)$ & $0.03805(50)$ & $0.01794(15)$ & $0.0055452(50)$ \\
6.950 & 221.8 & $2.0332765(52)$ & $0.0010256(87)$ & $0.0191246(19)$ & $0.01785(44)$ & $0.01182(21)$ & $0.0069963(116)$ \\
7.030 & 239.1 & $2.0009567(52)$ & $0.0008889(70)$ & $0.0171719(16)$ & $0.00732(49)$ & $0.00655(12)$ & $0.0088725(130)$ \\
7.150 & 267.4 & $1.9549570(41)$ & $0.0007594(33)$ & $0.01496641(85)$ & $0.002826(227)$ & $0.002693(65)$ & $0.0118853(116)$ \\
7.280 & 301.3 & $1.9081112(43)$ & $0.0006518(22)$ & $0.01296143(67)$ & $0.000578(120)$ & $0.001005(28)$ & $0.0155783(205)$ \\
7.373 & 327.8 & $1.8763272(51)$ & $0.0005648(11)$ & $0.01125795(32)$ & $0.000239(83)$ & $0.000522(29)$ & $0.0183751(154)$ \\
7.596 & 400.0 & $1.8052323(39)$ & $0.00044450(86)$ & $0.008876950(228)$ & $0.0000210(201)$ & $0.0001413(88)$ & $0.0256495(265)$ \\
7.825 & 488.9 & $1.7387004(82)$ & $0.00035425(5)$ & $0.007080600(105)$ & $0.00000040(14)$ & $0.0000461(10)$ & $0.0337466(261)$ \\
\hline
\end{tabular}

Table XI: Expectation value of local observables calculated on $N_{\tau}=10$ lattices 


\begin{tabular}{|c|l|l|l|l|l|l|l|}
\hline$\beta$ & $T[\mathrm{MeV}]$ & \multicolumn{1}{c|}{$\left\langle s_{G}\right\rangle$} & \multicolumn{1}{c|}{$\langle\bar{\psi} \psi\rangle_{l}$} & \multicolumn{1}{c|}{$\langle\bar{\psi} \psi\rangle_{s}$} & \multicolumn{1}{c|}{$\chi_{l}^{\text {disc }}$} & \multicolumn{1}{c|}{$\chi_{s}^{\text {disc }}$} & \multicolumn{1}{c|}{$L_{\text {bare }}$} \\
\hline 6.740 & 151.2 & $2.1257086(54)$ & $0.004079(97)$ & $0.0269239(25)$ & $0.4498(119)$ & $0.02317(53)$ & $0.0004772(73)$ \\
6.800 & 160.2 & $2.0981866(76)$ & $0.003016(120)$ & $0.0246617(30)$ & $0.5714(211)$ & $0.02569(175)$ & $0.0007167(44)$ \\
6.880 & 172.9 & $2.0629734(44)$ & $0.001882(75)$ & $0.0218834(45)$ & $0.3838(75)$ & $0.02479(102)$ & $0.0011690(73)$ \\
6.950 & 184.8 & $2.0334633(59)$ & $0.001339(52)$ & $0.0198904(40)$ & $0.1644(30)$ & $0.02098(123)$ & $0.0016813(86)$ \\
7.030 & 199.2 & $2.0011122(72)$ & $0.001025(22)$ & $0.0177901(23)$ & $0.0617(15)$ & $0.01489(61)$ & $0.0024077(75)$ \\
7.150 & 222.8 & $1.9550761(46)$ & $0.000808(10)$ & $0.0153880(17)$ & $0.0183(10)$ & $0.00823(55)$ & $0.0037030(71)$ \\
7.280 & 251.1 & $1.9082143(37)$ & $0.000675(13)$ & $0.0132415(21)$ & $0.0061(12)$ & $0.00312(13)$ & $0.0054060(154)$ \\
7.373 & 273.1 & $1.8764082(49)$ & $0.0005792(88)$ & $0.01146391(89)$ & $0.0042(12)$ & $0.002445(51)$ & $0.0068171(125)$ \\
7.596 & 333.3 & $1.8052889(42)$ & $0.00045065(76)$ & $0.00899511(28)$ & $0.000093(58)$ & $0.000682(11)$ & $0.0106861(146)$ \\
7.825 & 407.4 & $1.7387435(49)$ & $0.000358140(90)$ & $0.00715650(16)$ & $0.000000601(50)$ & $0.0001120(91)$ & $0.0153196(362)$ \\
\hline
\end{tabular}

Table XII: Expectation value of local observables calculated on $N_{\tau}=12$ lattices 
[1] K. G. Wilson, Phys. Rev. D10, 2445 (1974).

[2] M. Creutz, Phys.Rev. D21, 2308 (1980).

[3] J. Engels, F. Karsch, H. Satz, and I. Montvay, Phys. Lett. B101, 89 (1981).

[4] Y. Aoki, S. Borsanyi, S. Durr, Z. Fodor, S. D. Katz, et al., JHEP 0906, 088 (2009), 0903.4155.

[5] A. Bazavov, T. Bhattacharya, M. Cheng, C. DeTar, H. Ding, et al., Phys. Rev. D85, 054503 (2012), 1111.1710 .

[6] S. Borsanyi, Z. Fodor, S. D. Katz, S. Krieg, C. Ratti, et al., JHEP 1201, 138 (2012), 1112.4416.

[7] A. Bazavov et al. (HotQCD Collaboration), Phys.Rev. D86, 034509 (2012), 1203.0784.

[8] A. Bazavov, H. T. Ding, P. Hegde, O. Kaczmarek, F. Karsch, et al. (2014), 1404.6511.

[9] P. Petreczky, J. Phys. G39, 093002 (2012), 1203.5320.

[10] O. Philipsen, Prog. Part. Nucl. Phys. 70, 55 (2013), 1207.5999.

[11] C. DeTar and U. Heller, Eur.Phys.J. A41, 405 (2009), 0905.2949.

[12] A. D. Linde, Phys. Lett. B96, 289 (1980).

[13] P. Braun-Munzinger, K. Redlich, and J. Stachel (2003), to appear in Quark Gluon Plasma 3, eds. R.C. Hwa and Xin-Nian Wang, World Scientific Publishing, nuclth/0304013.

[14] S. Ejiri, F. Karsch, and K. Redlich, Phys.Lett. B633, 275 (2006), hep-ph/0509051.

[15] C. Gale, S. Jeon, and B. Schenke, Int.J.Mod.Phys. A28, 1340011 (2013), 1301.5893.

[16] T. Umeda et al. (WHOT-QCD Collaboration), Phys.Rev. D85, 094508 (2012), 1202.4719.

[17] U. M. Heller, F. Karsch, and B. Sturm, Phys. Rev. D60, 114502 (1999), hep-lat/9901010.

[18] T. Blum, C. E. Detar, S. A. Gottlieb, K. Rummukainen, U. M. Heller, et al., Phys. Rev. D55, 1133 (1997), heplat/9609036.

[19] K. Orginos, D. Toussaint, and R. Sugar (MILC Collaboration), Phys. Rev. D60, 054503 (1999), heplat/9903032.

[20] F. Karsch, E. Laermann, and A. Peikert, Phys. Lett. B478, 447 (2000), hep-lat/0002003.

[21] C. Bernard, T. Burch, C. E. DeTar, S. Gottlieb, L. Levkova, et al., Phys. Rev. D75, 094505 (2007), heplat/0611031.

[22] M. Cheng, N. Christ, S. Datta, J. van der Heide, C. Jung, et al., Phys. Rev. D77, 014511 (2008), 0710.0354.

[23] A. Bazavov, T. Bhattacharya, M. Cheng, N. Christ, C. DeTar, et al., Phys. Rev. D80, 014504 (2009), 0903.4379.

[24] M. Cheng, S. Ejiri, P. Hegde, F. Karsch, O. Kaczmarek, et al., Phys. Rev. D81, 054504 (2010), 0911.2215.

[25] S. Borsanyi, G. Endrodi, Z. Fodor, A. Jakovac, S. D. Katz, et al., JHEP 1011, 077 (2010), 1007.2580.

[26] S. Borsanyi, Z. Fodor, C. Hoelbling, S. D. Katz, S. Krieg, et al., Phys.Lett. B370, 99 (2014), 1309.5258.

[27] E. Follana et al. (HPQCD Collaboration, UKQCD Collaboration), Phys. Rev. D75, 054502 (2007), heplat/0610092.

[28] A. Bazavov and P. Petreczky, Phys.Rev. D87, 094505 (2013), 1301.3943.

[29] P. Cea, L. Cosmai, and A. Papa (2014), 1403.0821.
[30] A. Bazavov, H. Ding, P. Hegde, O. Kaczmarek, F. Karsch, et al., Phys. Rev. Lett. 109, 192302 (2012), 1208.1220 .

[31] A. Bazavov, H. T. Ding, P. Hegde, O. Kaczmarek, F. Karsch, et al., Phys. Rev. Lett. 111, 082301 (2013), 1304.7220 .

[32] A. Bazavov, H. T. Ding, P. Hegde, F. Karsch, C. Miao, et al. (2013), 1309.2317.

[33] A. Bazavov and P. Petreczky, Eur.Phys.J. A49, 85 (2013), 1303.5500.

[34] S. Kim, P. Petreczky, and A. Rothkopf (2013), 1310.6461.

[35] P. Petreczky (HotQCD Collaboration), PoS LATTICE2012, 069 (2012), 1211.1678.

[36] A. Bazavov (HotQCD Collaboration), Nucl.Phys.A904905 2013, 877c (2013), 1210.6312.

[37] A. Bazavov and P. Petreczky (HotQCD collaboration), J. Phys. Conf. Ser. 230, 012014 (2010), 1005.1131.

[38] R. Sommer, Nucl. Phys. B411, 839 (1994), heplat/9310022.

[39] C. Bernard et al. (MILC Collaboration), Phys. Rev. D71, 034504 (2005), hep-lat/0405029.

[40] A. Bazavov et al. (MILC Collaboration), PoS LATTICE2010, 074 (2010), 1012.0868.

[41] S. Borsanyi, S. Durr, Z. Fodor, C. Hoelbling, S. D. Katz, et al., JHEP 1209, 010 (2012), 1203.4469.

[42] P. Petreczky, Nucl. Phys. A830, 11C (2009), 0908.1917.

[43] F. Karsch, K. Redlich, and A. Tawfik, Eur. Phys. J. C29, 549 (2003), dedicated to Rolf Hagedorn, hep-ph/0303108.

[44] M. Cheng, P. Hendge, C. Jung, F. Karsch, O. Kaczmarek, et al., Phys. Rev. D79, 074505 (2009), 0811.1006.

[45] P. Huovinen and P. Petreczky, Nucl. Phys. A837, 26 (2010), 0912.2541.

[46] S. Borsanyi et al. (Wuppertal-Budapest Collaboration), JHEP 1009, 073 (2010), 1005.3508.

[47] J. Beringer et al. (Particle Data Group), Phys.Rev. D86, 010001 (2012).

[48] R Core Team, R: A Language and Environment for Statistical Computing (Vienna, Austria, 2013), URL http: //www.R-project.org/.

[49] W. N. Venables and B. D. Ripley, Modern Applied Statistics with $S$ (Springer, New York, 2002), 4th ed., iSBN 0-387-95457-0, URL http://www.stats.ox.ac.uk/pub/ MASS4.

[50] F. E. H. Jr, with contributions from Charles Dupont, and many others., Hmisc: Harrell Miscellaneous (2014), r package version 3.14-4, URL http://CRAN.R-project. org/package=Hmisc.

[51] C. Hung and E. V. Shuryak, Phys.Rev.Lett. 75, 4003 (1995), hep-ph/9412360.

[52] J. Engels and F. Karsch, Phys. Rev. D85, 094506 (2012), 1105.0584.

[53] N. Haque, A. Bandyopadhyay, J. O. Andersen, M. G. Mustafa, M. Strickland, et al., JHEP 1405, 027 (2014), 1402.6907.

[54] M. Laine and Y. Schroder, Phys.Rev. D73, 085009 (2006), hep-ph/0603048.

[55] M. Clark, A. Kennedy, and Z. Sroczynski, Nucl. Phys. Proc. Suppl. 140, 835 (2005), hep-lat/0409133.

[56] M. Hasenbusch, Phys.Lett. B519, 177 (2001), heplat/0107019.

[57] A. Bazavov et al. (MILC collaboration), Phys. Rev. D82, 
074501 (2010), 1004.0342.

[58] C. R. Allton, Nucl.Phys.Proc.Suppl. 53, 867 (1997), heplat/9610014.

[59] A. Bazavov et al. (The MILC Collaboration) (2013), 1311.1474

[60] C. Davies, E. Follana, I. Kendall, G. Lepage, and C. Mc-
Neile (HPQCD Collaboration), Phys. Rev. D81, 034506 (2010), 0910.1229.

[61] S. Aoki, Y. Aoki, C. Bernard, T. Blum, G. Colangelo, et al. (2013), 1310.8555 . 